hep-th/0011047

MRI-P-001101

\title{
Unstable Non-BPS D-Branes of Type-II String Theories in Light-Cone Green-Schwarz Formalism
}

\author{
Partha Mukhopadhyay由 \\ Harish-Chandra Research Institute \\ Chhatnag Road, Jhusi, Allahabad 211019, India
}

\begin{abstract}
The problem of describing the boundary states of unstable non-BPS D-branes of type-II string theories in light-cone Green-Schwarz (GS) formalism is addressed. Regarding the type II theories in light-cone gauge as different realizations of the $\hat{S O}(8)_{k=1}$ Kac-Moody algebra, the non-BPS D-brane boundary states of these theories are given in terms of the relevant Ishibashi states constructed in this current algebra. Using the expressions for the current modes in terms of the GS variables it is straightforward to reexress the boundary states in the GS formalism. The problem that remains is the lack of manifest $S O(8)$ covariance in these expressions. We also derive the various known expressions for the BPS and non-BPS D-brane boundary states by starting with the current algebra Ishibashi states.
\end{abstract}

\footnotetext{
${ }^{1}$ partha@mri.ernet. in

${ }^{2}$ Formerly Mehta Research Institute of Mathematics \& Mathematical Physics
} 


\section{Contents}

\begin{tabular}{llr}
\hline & Introduction and Summary & 2
\end{tabular}

2 The Affine $\hat{S O}(8)_{k=1}$ Current Algebra and its Irreducible Unitary Highest $\begin{array}{ll}\text { Weight Representations } & 6\end{array}$

3 Realization of $\hat{S O}(8)_{k=1}$ in terms of NSR, GS and Bosonic Fields 9

4 Ishibashi States and Type IIB D-Branes $\quad 14$

4.1 Ishibashi States . . . . . . . . . . . . . . . . . . . . . . . . 14

4.2 Type IIB D-Branes . . . . . . . . . . . . . . . . . . . . . . . . . . 15

5 Basis Construction and the Non-BPS Boundary States 21

5.1 Basis Construction . . . . . . . . . . . . . . . . . 21

5.2 Non-BPS Boundary States . . . . . . . . . . . . . . . . . . . . . . . . . . 24

\begin{tabular}{|ll}
\hline A Vacuum Representations & 31
\end{tabular}

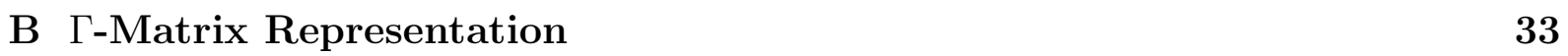

\begin{tabular}{|l|l|}
\hline C Norm of Bosonic Vacuum states & 34 \\
\hline
\end{tabular}

\section{Introduction and Summary}

The ten dimensional type IIA and IIB superstring theories admit BPS $D p$-branes [1, 2] for even and odd $p$ respectively. The restriction on $p$ comes from the condition that half of the supersymmetries (SUSY) of the bulk be preserved in the world-volume theory. It has been realized recently that IIA and IIB theories possess D-branes of respectively odd and even dimensions also, which are called non-BPS D-branes [3, 4, 5] (for reviews and complete list of references see [6, []), as they break all the SUSY of the bulk. As it is well known that string theory has two types of formalisms, namely NSR (Neveu-Schwarz-Ramond) [9] and GS (Green-Schwarz) 10 formalisms, it is desirable to have descriptions of all these nonperturbative objects in both the formalisms. This is because, in different situations different formalisms become useful. In most of the situations (at least in the flat space), NSR formalism has been useful where we have proper description of all the D-branes in terms of the NSR variables (see [1, 2, 3, 4, 5, 6, 7, 9, 12, 13]). One specific use of the GS description would be to check SUSY of a D-brane. Since the SUSY generators 10, 15 are simple in terms of the GS fields, doing this would be easier if the D-brane boundary state 12] (for D-brane boundary states in light-cone gauge see [3, 7, 13, 14, 15]) is written in terms of the GS variables. The boundary states for the BPS D-branes in GS formalism have already been found by Green[14] and Green and Gutperle[15]. In this paper we 
address the problem of describing the unstable non-BPS D-branes in ten flat dimensions in light-cone GS formalism [10]. More specifically we will concentrate only on the fermionic part of the world-sheet theory as the bosonic part is the same in the two formalisms. Throughout this paper the phrase "non-BPS branes" would imply the unstable non-BPS D-branes of IIA and IIB string theories in ten dimensional Minkowski space.

Let us first discuss the problem without going into the technical details. A D-brane has two different descriptions - open string world-volume description and closed string boundary state description. In the first approach, one puts proper open string boundary conditions on the fields living on the fundamental string world-sheet. The tangential and transverse directions to the brane have respectively Neumann and Dirichlet boundary conditions. A space-time parity transformation along a single direction only on the right moving (antiholomorphic) variables interchanges the Neumann and Dirichlet boundary conditions along that direction [8, 9]. Starting from any BPS boundary condition in a given theory one generates all the non-BPS boundary conditions by applying all possible parity transformations involving only odd number of directions. Since the world-sheet fermions in NSR formalism form a vector representation of the space-time group $S O(9,1)^{\text {f }}$, they transform in a simple way under parity. Hence in these variables the boundary conditions for non-BPS D-branes are as simple as those for BPS branes, and the conformal field theory on the world-sheet has a simple description. But the world-sheet fermions in the light-cone GS formalism form spinor representations of $S O(8)$. A parity transformation along an odd number of directions converts a spinor representation to a conjugate spinor representation [16] which does not belong to the set of variables used for defining the theory. This complication makes it difficult to describe the boundary conformal field theory for a non-BPS D-brane in GS formalism. Because of the same difficulty, the usual procedure of converting the open string boundary conditions to the closed string gluing conditions and then solving that in the form of a boundary state, does not work.

One way to solve the problem may seem to translate the known non-BPS boundary state in NSR formalism in terms of the GS variables. But the field redefinition required to go from the NSR variables to the GS variables is too complicated to do this. Thus we need to follow a different approach. For this let us try to understand the problem described, in a more fundamental way. The reason that we have different formalisms like NSR and GS of the same theory (type IIA or IIB) [10, [1] is the existence of an underlying local current algebra[17, [18], namely $\hat{S O}(8)_{k=1}$ and the crucial property of the group $S O(8)$ that it has three eight dimensional representations, namely the vector, spinor and conjugate spinor representations][16]. This makes it possible to formulate a realization of the $\hat{S O}(8)_{k=1}$ current algebra in different languages 17 like that of NSR and GS, where in the first case the world-sheet fermions form the vector representation and in the second case spinor and/or conjugate spinor representation. From this point of view the problem at hand is

\footnotetext{
${ }^{3}$ In light-cone gauge this comes down to $S O(8)$.

${ }^{4}$ See ref.[10 for a discussion on 'triality', which is a symmetry of the $S O(8)$ Dynkin diagram under which these three fundamental and inequivalent representations tranform among each other.
} 
that of expressing the boundary state of a non-BPS D-brane in a specific realization (GS) of the current algebra $\hat{S O}(8)_{k=1}$. Our strategy will be to analyse the D-brane boundary states in these theories in terms of the current algebra itself [19, 20. Although going from one formalism to another is in general complicated, expressing the currents in any formalism is straightforward. Thus once we get a boundary state in terms the local $S O(8)$ currents, that can be reexpressed in the GS formalism.

We proceed along the following steps:

1. We consider $S O(8)$ Kac-Moody current algebra of level $k=1$ associated with the NSR/GS fermions and its irreducible unitary highest weight representations (irreps) [17. The type IIA and IIB theories are regarded as different realizations of the same current algebra. These two theories differ by the irreps that are realized.

2. Given a consistent boundary condition on the currents, one can construct a unique Ishibashi state [19, 20] for each irrep. Therefore given a boundary condition relevant for a specific D-brane, there is an Ishibashi state for each irrep of $\hat{S O}(8)_{k=1}$. Not all the Ishibashi states are important for the present purpose. Only those, the corresponding irreps for which are realized in a given theory, are needed to construct the boundary states for D-branes of that theory. It turns out that if the boundary condition is BPS in a given theory then there are two realizable Ishibashi states and if it is non-BPS then there is only one. In the first case the two Ishibashi states are the NS-NS and the R-R parts of the corresponding D-brane boundary state and in the second case the Ishibashi state itself is the non-BPS boundary state.

3. To write down an Ishibashi state explicitly one needs to consider a complete set of basis vectors for the Hilbert space [20]. One can construct various sets of basis states. It can be constructed in a given formalism by applying the corresponding negative mode fermionic oscillators on the ground state(s). This is the natural fermionic oscillator basis in that formalism. The basis can also be constructed purely in terms of the current modes, which is independent of any formalism. We will show that in a given formalism, choosing the fermionic oscillator basis to work with, directly reproduces the standard form of the already known boundary states. This means that the boundary states for both the BPS and non-BPS D-branes in the NSR formalism and only the BPS D-branes in the GS formalism can be obtained in this way. We will see that in the current algebraic language the present problem can be stated by saying that the natural fermionic oscillator basis arising in the GS formalism is not suitable for writing down the Ishibashi states corresponding to the non-BPS D-branes

\footnotetext{
${ }^{5}$ Actually the Ishibashi states considered themselves do not construct the complete D-brane boundary states, but rather form only the fermionic oscillator part of that. We will make it more precise in subsec. 4.2 .

${ }^{6}$ This is related to the fact that implementation of the non-BPS boundary conditions on the spinor variables is not as easy as it is in the case of variables transforming as vectors.
} 
4. We will argue that it is preferable to construct the basis in terms of the current modes for writing down the Ishibashi states corresponding to the non-BPS D-branes. Since the currents transform as tensors under parity tranformation, implementation of both the BPS and non-BPS boundary conditions is quite straightforward. But there is a principal drawback of this type of basis. The free module constructed out of the current modes contains null states as opposed to the case of various fermionic oscillator basis. To use it to construct the Ishibashi states one needs to make the basis free of null states. This can be done, but one loses explicit covariance during this process. Given this basis, one can reexpress this in terms of the Green-Schwarz variables, but the resulting expression is not manifestly $S O(8)$ covariant. The lack of manifest covariance is the principal drawback of this approach.

Organization of the paper: In Sec. 2 we briefly discuss the affine $\hat{S O}(8)_{k=1}$ current algebra both in the covariant and the Cartan-Weyl basis and quote the basic results about its irreps which will be needed for the present purpose. In Sec. 3 we discuss how $\hat{S O}(8)_{k=1}$ is realized in NSR, GS and bosonic formalism. Here the 'bosonic formalism' considered] is the one which is obtained by performing the abelian bosonization] 21] of the NSR or the GS fermions suitably on the world-sheet. These sections establish the notations and the basic definitions for the various variables that we will work with. In Sec. Twe introduce the current algebra Ishibashi states, and demonstrate how using the present current algebraic approach one can derive the known results for BPS and nonBPS D-brane boundary states by constructing the basis states in terms of the appropriate fermionic oscillators. At this point, we indicate the problem of using the similar basis for constructing the boundary states for non-BPS D-branes in the GS formalism. In Sec. 5 we give the explicit construction of an orthonormal basis in terms of the current modes and then show how to get the non-BPS D-brane boundary states in GS formalism by using this basis. The appendices contain some additional technical details. Appendix A contains the explicit construction of the current algebraic vacuum representations on which the irreps are built up by applying the current modes. The specific representation chosen for the $S O(8)$ gamma matrices is given in appendix $\mathbb{B}$. Appendix $\mathrm{O}$ shows that the bosonic vacuum states defined in Sec. 5 are normalised to 1, which supports the proof (given in Sec. 5) of orthonormality of the constructed basis.

\footnotetext{
${ }^{7}$ It is not true that in principle one needs to cosider the bosonic formalism in the present approach. We need to consider it because of the following reason: the non-covariant basis that we will construct in terms of the current modes will have exact resemblance (quantum number wise) with the natural basis that one would construct in the bosonic formalism.

8 Throughout this paper we will use the word 'bosons' to refer only the abelian bosons. The nonabelian bosons will always be called 'currents'.
} 


\section{The Affine $\hat{S O}(8)_{k=1}$ Current Algebra and its Irre- ducible Unitary Highest Weight Representations}

The affine Kac-Moody algebras, their representations, realizations in terms of fermionic and bosonic fields and specifically superstring theory in the context of $\hat{S O}(8)_{k=1}$ have been discussed quite extensively and in a self-contained manner in ref. [17]. The purpose of this section is to introduce notations, basic definitions and review results only for $\hat{S O}(8)_{k=1}$, which we will need for the present paper.

The infinite dimensional Lie algebra $\hat{S O}(8)_{k=1}$ is given by:

$$
\begin{aligned}
{\left[J_{m}^{\mu \nu}, J_{n}^{\rho \sigma}\right]=} & i\left(\delta^{\nu \rho} J_{(m+n)}^{\mu \sigma}+\delta^{\mu \sigma} J_{(m+n)}^{\nu \rho}-\delta^{\mu \rho} J_{(m+n)}^{\nu \sigma}-\delta^{\nu \sigma} J_{(m+n)}^{\mu \rho}\right) \\
& +m \delta_{(m+n), 0}\left(\delta^{\mu \rho} \delta^{\nu \sigma}-\delta^{\mu \sigma} \delta^{\nu \rho}\right)
\end{aligned}
$$

where $J_{n}^{\mu \nu}=-J_{n}^{\nu \mu}(\mu, \nu=1,2, \ldots, 8, n \in \mathbf{Z})$ are the elements of the algebra. We define the Cartan-Weyl (CW) basis as follows:

$$
\begin{gathered}
H_{n}^{i}=J_{n}^{2 i-1,2 i}, \quad(i=1, . ., 4), \\
E_{n}^{\alpha}=\frac{1}{2}\left[\left(J_{n}^{2 i-1,2 j-1}-\eta_{1} \eta_{2} J_{n}^{2 i, 2 j}\right)-i\left(\eta_{1} J_{n}^{2 i, 2 j-1}+\eta_{2} J_{n}^{2 i-1,2 j}\right)\right]_{i<j},
\end{gathered}
$$

where $\alpha=\left(\eta_{1} e_{i}+\eta_{2} e_{j}\right)_{i<j}$ is a nonzero root, $\eta_{1}, \eta_{2}= \pm 1$ and $e_{i}$ 's are the orthonormal basis of the Cartan subalgebra (CSA) space:

$$
e_{1}=\left(\begin{array}{l}
1 \\
0 \\
0 \\
0
\end{array}\right), \quad e_{2}=\left(\begin{array}{l}
0 \\
1 \\
0 \\
0
\end{array}\right), \quad e_{3}=\left(\begin{array}{l}
0 \\
0 \\
1 \\
0
\end{array}\right), \quad e_{4}=\left(\begin{array}{l}
0 \\
0 \\
0 \\
1
\end{array}\right) \text {. }
$$

In the CW basis the algebra-elements are the twenty-four $E_{n}^{\alpha}$ 's and four $H_{n}^{i}$ 's $(\forall n \in \mathbf{Z})$, in terms of which the algebra takes the following form:

$$
\begin{aligned}
& {\left[H_{m}^{i}, H_{n}^{j}\right]=} m \delta^{i j} \delta_{m+n, 0}, \\
& {\left[H_{m}^{i}, E_{n}^{\alpha}\right]=}\left(\alpha \cdot e_{i}\right) E_{(m+n)}^{\alpha}, \\
& {\left[E_{m}^{\alpha}, E_{n}^{\beta}\right]= \begin{cases}\epsilon(\alpha, \beta) E_{(m+n)}^{\alpha+\beta} & \text { if }(\alpha+\beta) \text { is a root }, \\
\left(\alpha . e_{i}\right) H_{(m+n)}^{i}+m \delta_{m+n, 0} & \text { if } \alpha=-\beta=-1, \\
& \text { i.e. } \alpha \cdot \beta=-2, \\
0 & \text { otherwise },\end{cases} }
\end{aligned}
$$


Here $\epsilon(\alpha, \beta)$ is a phase antisymmetric in $\alpha$ and $\beta$ when $\alpha . \beta=-1$ and can be computed explicitly using eqns. (2.1) and (2.3). The zero-modes elements satisfy the finite dimensional $S O(8)$ algebra. Out of the twenty-four non-zero roots the following are the twelve positive roots:

$$
\begin{aligned}
& \alpha_{1}=\psi=e_{1}+e_{2}, \quad \alpha_{2}=\alpha_{1}^{s}=e_{1}-e_{2}, \quad \alpha_{3}=\alpha_{4}^{s}=e_{3}+e_{4}, \\
& \alpha_{4}=\alpha_{3}^{s}=e_{3}-e_{4}, \quad \alpha_{5}=e_{1}+e_{3}, \quad \alpha_{6}=e_{1}-e_{3}, \\
& \alpha_{7}=e_{1}+e_{4}, \quad \alpha_{8}=e_{1}-e_{4}, \quad \alpha_{9}=e_{2}+e_{3}, \\
& \alpha_{10}=\alpha_{2}^{s}=e_{2}-e_{3}, \quad \alpha_{11}=e_{2}+e_{4}, \quad \alpha_{12}=e_{2}-e_{4} \text {. }
\end{aligned}
$$

where $\psi$ is the highest root and $\alpha_{i}^{s}(i=1, \ldots, 4)$ are the simple roots. Now we define various conformal fields (local currents) on the $z$-plane with the algebra elements as their modes:

$$
\mathcal{J}(z)=\sum_{n \in \mathbf{Z}} \frac{\mathcal{J}_{n}}{z^{n+1}}
$$

where $\mathcal{J}$ stands for any of $J^{\mu \nu}, H^{i}$ and $E^{\alpha}$. We connect the $z$-plane with the closed string tree world-sheet (cylinder) of the type II theories by the conformal map $z=e^{t} e^{-i \sigma}$, where $(t, \sigma)$ parameterize the cylinder with the range: $-\infty<t<\infty, 0 \leq \sigma<2 \pi$. Sugawara's construction for the energy-momentum tensor reads 17, 18, 9]:

$$
\begin{aligned}
T^{S U G}(z) & =\frac{1}{14}: J J:(z), \\
& =\sum_{n \in \mathbf{Z}} \frac{L_{n}^{S U G}}{z^{n+2}},
\end{aligned}
$$

where, the factor of 14 comes from $2 k+C_{2}, C_{2}$ being the quadratic Casimir in the adjoint representation. For $S O(8), C_{2}=12$. The composite object : $J J:(z)$ has the following definition[9]:

$$
: J J:(z)=\lim _{w \rightarrow z}\left(\sum_{\mu<\nu} J^{\mu \nu}(z) J^{\mu \nu}(w)-\frac{28}{(z-w)^{2}}\right) .
$$

The modes $L_{n}^{S U G}$ satisfy a Virasoro algebra with central charge $c=4$.

\section{Irreducible Unitary Highest Weight Representations:}

Here we will discuss the results of the unitary highest weight representations which are irreducible under the Kac-Moody algebra (2.1). A highest weight state $|w\rangle$ for a given weight vector $w=\sum_{i}(w)_{i} e_{i}$ is defined in the following way:

$$
\begin{aligned}
E_{0}^{\alpha}|w\rangle & =E_{n}^{ \pm \alpha}|w\rangle=0, & & \forall n>0, \forall \alpha>0, \\
H_{n}^{i}|w\rangle & =0, & & \forall n>0, \forall i, \\
H_{0}^{i}|w\rangle & =(w)_{i}|w\rangle . & &
\end{aligned}
$$


This state satisfies: $L_{0}^{S U G}|w\rangle=h_{w}|w\rangle$ where $h_{w}=w^{2} / 2>0$. An irreducible representation labelled by $w$ is obtained by applying $J_{-n}^{\mu \nu} \quad(n \geq 0)$ on $|w\rangle$ in all possible ways. One checks unitarity i.e. the absence of the negative norm states with respect to the usual hermitian inner product. The representation obtained in this way contains null states which one has to put to zero by hand. For a unitary representation, the four dimensional vector defined by the $H_{0}^{i}(i=1, \ldots, 4)$ eigenvalues of any state in the representation lies on a lattice $\Lambda_{W}$ called weight lattice which is dual to the root lattice $\Lambda_{R}$ generated by all the nonzero roots. Since any root can be expanded in terms of the simple roots with integer coefficients, $\alpha_{i}^{s}$ 's can be taken as the basis for $\Lambda_{R}$. It follows from the definition of a lattice being dual to another, that $\Lambda_{W}$ can be generated by certain vectors $f_{i}$ called the fundamental weights defined by,

$$
f_{i} \cdot \alpha_{j}^{s}=\delta_{i j}, \quad 1 \leq i, j \leq 4 .
$$

Given eqns. (2.8) it is easy to obtain the following four $S O(8)$ fundamental weights:

$$
f_{1}=e \equiv e_{1}=\left(\begin{array}{l}
1 \\
0 \\
0 \\
0
\end{array}\right), f_{2}=\psi=\left(\begin{array}{l}
1 \\
1 \\
0 \\
0
\end{array}\right), f_{3}=\bar{\delta} \equiv \frac{1}{2}\left(\begin{array}{c}
1 \\
1 \\
1 \\
-1
\end{array}\right), f_{4}=\delta \equiv \frac{1}{2}\left(\begin{array}{l}
1 \\
1 \\
1 \\
1
\end{array}\right) \text {. }
$$

Now it can be shown that the value of $k$ restricts the highest weights that can occur as labels of the representations. For $\hat{S O}(8)_{k=1}$, there are four irreducible representations corresponding to the follwing highest weights:

$$
w=0, \quad e, \quad \bar{\delta}, \quad \delta .
$$

It is easy to show that,

$$
\Lambda_{W}=\Lambda_{R} \sqcup \Lambda_{e} \sqcup \Lambda_{\bar{\delta}} \sqcup \Lambda_{\delta},
$$

where $\sqcup \equiv$ union of disjoint sets and $\Lambda_{w} \equiv w+\Lambda_{R} \quad(w=e, \bar{\delta}, \delta)$.

Each highest weight representation corresponding to a weight $w$ has a finite dimensional subspace of states which are annihilated by $J_{n}^{\mu \nu} \forall n>0$. These form a representation of the zero mode subalgebra of the current algebra and will be called the vacuum representation corresponding to the given highest weight representation. If $n_{(w)}$ denotes the dimension of the vacuum representation, we lable these states as $\left|\Pi_{0}^{(w)}, j\right\rangle, j=1, \ldots, n_{(w)}$. These satisfy the following ptoperty:

$$
J_{0}^{\mu \nu}\left|\Pi_{0}^{(w)}, j\right\rangle=\left(T_{(w)}^{\mu \nu}\right)_{l j}\left|\Pi_{0}^{(w)}, l\right\rangle,
$$

where $T_{(w)}^{\mu \nu}$ are $n_{(w)} \times n_{(w)}$ hermitian matrices ? representing the current zero-modes in the vacuum representation and repeated indices are summed over. The $S O(8)$ group elements

\footnotetext{
${ }^{9}$ For $S O(8)$ it is possible to impose the reality property on the representations. This makes the matrices $T_{(w)}^{\mu \nu}$ purely imaginary and antisymmetric.
} 
are parameterized in the following way:

$$
\mathcal{O}(\theta)=\exp \left(\frac{i}{2} \theta_{\mu \nu} J_{0}^{\mu \nu}\right)
$$

where $\theta_{\mu \nu}=-\theta_{\nu \mu}, \theta_{\mu \nu}^{*}=\theta_{\mu \nu}$. Clearly the vacuum states are created by applying the current zero-modes on the highest weight state. The explicit matrix representations for the four vacuum representations of $\hat{S O}(8)_{k=1}$ are given in appendix $\mathrm{A}$.

\section{Realization of $\hat{S O}(8)_{k=1}$ in terms of NSR, GS and Bosonic Fields}

The Euclidean actions in the NSR and GS formalism take the following form on the z-plane:

$$
\begin{aligned}
S_{N S R} & =\frac{1}{4 \pi} \int d^{2} z\left(\Psi^{\mu} \bar{\partial} \Psi^{\mu}+\tilde{\Psi}^{\mu} \partial \tilde{\Psi}^{\mu}\right) \\
S_{G S}^{I I B} & =\frac{1}{4 \pi} \int d^{2} z\left(S^{a} \bar{\partial} S^{a}+\tilde{S}^{a} \partial \tilde{S}^{a}\right), \\
S_{G S}^{I I A} & =\frac{1}{4 \pi} \int d^{2} z\left(S^{a} \bar{\partial} S^{a}+\tilde{S}^{\dot{a}} \partial \tilde{S}^{\dot{a}}\right) .
\end{aligned}
$$

$\Psi^{\mu}(z)$ and $\tilde{\Psi}^{\mu}(\bar{z})$ transform as the states $|\mu\rangle, S^{a}(z), \tilde{S}^{a}(\bar{z})$ as $|a\rangle$ and $\tilde{S}^{\dot{a}}(\bar{z})$ as $|\dot{a}\rangle$ under $S O(8)$ with the transformation laws defined in appendix A. All the holomorphic fields have conformal dimension $\left(\frac{1}{2}, 0\right)$ and antiholomorphic fields have conformal dimension $\left(0, \frac{1}{2}\right)$. The mode expansions are:

$$
\Psi^{\mu}(z)=\sum_{r \in \mathbf{Z}+\nu} \frac{\Psi_{r}^{\mu}}{z^{r+1 / 2}}, \quad \tilde{\Psi}^{\mu}(\bar{z})=\sum_{r \in \mathbf{Z}+\tilde{\nu}} \frac{\tilde{\Psi}_{r}^{\mu}}{\bar{z}^{r+1 / 2}},
$$

where $\nu, \tilde{\nu}=0, \frac{1}{2}$ corresponding to respectively $\mathrm{R}$ and NS sector.

$$
S^{a}(z)=\sum_{n \in \mathbf{Z}} \frac{S_{n}^{a}}{z^{n+1 / 2}}, \quad \tilde{S}^{a}(\bar{z})=\sum_{n \in \mathbf{Z}} \frac{\tilde{S}_{n}^{a}}{\bar{z}^{n+1 / 2}}, \quad \tilde{S}^{\dot{a}}(\bar{z})=\sum_{n \in \mathbf{Z}} \frac{\tilde{S}_{n}^{\dot{a}}}{\bar{z}^{n+1 / 2}} .
$$

The nontrivial anticommutation relations are:

$$
\left\{\Psi_{r}^{\mu}, \Psi_{s}^{\nu}\right\}=\delta^{\mu \nu} \delta_{r+s, 0}, \quad\left\{S_{m}^{a}, S_{n}^{b}\right\}=\delta^{a b} \delta_{m+n, 0}
$$

and similarly for the antiholomorphic modes. The OPE's are:

$$
\Psi^{\mu}(z) \Psi^{\nu}(0) \sim \frac{\delta^{\mu \nu}}{z}, \quad S^{a}(z) S^{b}(0) \sim \frac{\delta^{a b}}{z}
$$


and similarly for the antiholomorphic fields. Now we define the fermionic part of the energy-momentum tensors in NSR and GS formalism as the following:

$$
T^{N S R}(z)=-\frac{1}{2}: \Psi^{\mu} \partial \Psi^{\mu}:(z), \quad T^{G S}(z)=-\frac{1}{2}: S^{a} \partial S^{a}:(z)
$$

and similarly for the antiholomorphic part. For $\tilde{T}^{G S}(\bar{z})$ we have sum over $a$ or $\dot{a}$ depending upon whether it is IIB or IIA respectively. The conformal normal ordering of the fermionic fields are defined as follows:

$$
\begin{aligned}
& : \Psi^{\mu} \Psi^{\nu}(z):=\lim _{w \rightarrow z}\left[\Psi^{\mu}(w) \Psi^{\nu}(z)-\frac{\delta^{\mu \nu}}{(w-z)}\right] \\
& : S^{a} S^{b}(z):=\lim _{w \rightarrow z}\left[S^{a}(w) S^{b}(z)-\frac{\delta^{a b}}{(w-z)}\right] .
\end{aligned}
$$

Each of the above energy-momentum tensors has standard OPE with itself with central charge $c=4$, which is the same as that for $T^{S U G}$.

In addition to the NSR and GS formalisms we will consider another formalism, which is obtained through abelian bosonization 21] of the fermions. This is well known that the theory of the four world-sheet bosonic fields obtained by bosonizing the eight world-sheet fermions (either of NSR and GS type) is equivalent to the fermionic theory, if the bosonic momenta are properly quantized. The reason we cosider the bosonic formalism is that the basis of states in the Hilbert space that we will construct will have explicit resemblance with the natural basis that one would construct in the bosonic formalism. Here we will give the bosonization rules and the basic definitions in the bosonic formalism as we have done in the fermionic case. We start by defining the fermionic fields in the lattice basis:

$$
\begin{aligned}
\lambda^{ \pm e_{j}}(z) & \equiv \frac{1}{\sqrt{2}}\left(\Psi^{\mu=2 j-1}(z) \mp i \Psi^{\mu=2 j}(z)\right), \\
\chi^{ \pm \delta_{j}}(z) & \equiv \frac{1}{\sqrt{2}}\left(S^{a=2 j-1}(z) \mp i S^{a=2 j}(z)\right), \\
\tilde{\xi}^{ \pm \bar{\delta}_{j}}(\bar{z}) & \equiv \frac{1}{\sqrt{2}}\left(\tilde{S}^{\dot{a}=2 j-1}(\bar{z}) \mp i \tilde{S}^{\dot{a}=2 j}(\bar{z})\right) .
\end{aligned}
$$

and the other antiholomorphic fields namely $\tilde{\lambda}^{ \pm e_{i}}(\bar{z})$ and $\tilde{\chi}^{ \pm \delta_{i}}(\bar{z})$ are defined in the similar fashion. Then we bosonize in the following way:

$$
\begin{aligned}
& \lambda^{ \pm e_{i}}(z) \sim: \exp \left( \pm i e_{i} . \phi\right)(z): \\
& \chi^{ \pm \delta_{i}}(z) \sim: \exp \left( \pm i \delta_{i} . \phi\right)(z): \\
& \tilde{\xi}^{ \pm \bar{\delta}_{i}}(\bar{z}) \sim: \exp \left( \pm i \bar{\delta}_{i} . \tilde{\phi}\right)(\bar{z}):
\end{aligned}
$$

Bosonizations of the other antiholomorphic fields are similar to the corresponding holomorphic fields with $\phi(z)$ replaced by $\tilde{\phi}(\bar{z})$. The symbol $\sim$ in the above equations implies 
that the relations are true upto proper cocycle factors which need to satisfy various consistency conditions. We will not need the explicit form of the cocycle factors for our analysis and will always mention whenever the symbol $\sim$ means it 10 . The fields $\phi(z)$ and $\tilde{\phi}(\bar{z})$ are four component fields representing the CSA space. These fields are compactified in such a way that the compact momenta take values on $\Lambda_{W}$. The mode expansions for the bosonic fields and commutation relations for different modes can be shown to be as follows:

$$
\begin{gathered}
\phi_{i}(z) \equiv e_{i} . \phi(z)=\varphi^{i}-i P^{i} \ln z+i \sum_{n \neq 0} \frac{1}{n} \frac{\beta_{n}^{i}}{z^{n}} \\
{\left[\varphi^{i}, P^{j}\right]=i \delta^{i j}, \quad\left[\beta_{m}^{i}, \beta_{n}^{j}\right]=m \delta_{m+n, 0} \delta^{i j} .}
\end{gathered}
$$

The exponential operator in the bosonization rules has the standard definition in terms of the modes:

$$
: e^{i \rho . \phi}(z): \equiv e^{i \rho . \varphi} z^{\rho . P} e^{-\sum_{n<0} \frac{\rho . \beta_{n}}{n z^{n}}} e^{-\sum_{n>0} \frac{\rho . \beta_{n}}{n z^{n}}} .
$$

One can derive the OPE:

$$
\phi^{i}(z) \phi^{j}(0) \sim-\delta^{i j} \ln z .
$$

The energy-momentum tensor is defined as,

$$
T^{B O S}(z)=-\frac{1}{2}: \partial \phi^{i} \partial \phi^{i}:(z)
$$

where,

$$
: \partial \phi^{i} \partial \phi^{i}:(z)=\lim _{w \rightarrow z}\left[\partial_{w} \phi^{i}(w) \partial_{z} \phi^{i}(z)+\frac{4}{(w-z)^{2}}\right] .
$$

Again $T^{B O S}(z)$ has the usual OPE with itself with central charge $c=4$. For the right moving part there is a similar eqn. for each of the above eqns. (3.12) - (3.17). Now let us specify the $S O(8)$ currents in different formalisms:

\section{NSR:}

$$
J^{\mu \nu}(z)=\frac{i}{2} \Sigma_{\rho \sigma}^{\mu \nu}: \Psi^{\rho} \Psi^{\sigma}(z): \quad, \quad \tilde{J}^{\mu \nu}(\bar{z})=\frac{i}{2} \Sigma_{\rho \sigma}^{\mu \nu}: \tilde{\Psi}^{\rho} \tilde{\Psi}^{\sigma}(\bar{z}):
$$

where $\Sigma_{\rho \sigma}^{\mu \nu}=\delta^{\mu \rho} \delta^{\nu \sigma}-\delta^{\mu \sigma} \delta^{\nu \rho}$.

\footnotetext{
${ }^{10}$ If not mentioned, it will always carry its usual meaning, for example in case of OPE, it would imply that the relation is true up to a regular part.

${ }^{11}$ Upper and lower indices are indistinguishable for all the fundamental representations.
} 


\section{GS:}

$$
\begin{array}{ll}
J^{\mu \nu}(z)=\frac{i}{4}\left(\gamma^{\mu} \bar{\gamma}^{\nu}\right)_{a b}: S^{a} S^{b}(z): & \text { (IIB, IIA) } \\
\tilde{J}^{\mu \nu}(\bar{z})=\frac{i}{4}\left(\gamma^{\mu} \bar{\gamma}^{\nu}\right)_{a b}: \tilde{S}^{a} \tilde{S}^{b}(\bar{z}): & \text { (IIB) }, \\
\tilde{J}^{\mu \nu}(\bar{z})=\frac{i}{4}\left(\bar{\gamma}^{\mu} \gamma^{\nu}\right)_{\dot{a} \dot{b}}: \tilde{S}^{\dot{a}} \tilde{S}^{\dot{b}}(\bar{z}): & \text { (IIA) } .
\end{array}
$$

where $\gamma^{\mu}, \bar{\gamma}^{\mu}$ are $8 \times 8$ matrices defined in appendix $\mathrm{B}$.

\section{Bosonic:}

$$
\begin{array}{lll}
H^{i}(z)=i \partial \phi^{i}(z), & E^{\alpha}(z) \sim: e^{i \alpha . \phi}(z): \\
\tilde{H}^{i}(\bar{z})=i \bar{\partial} \tilde{\phi}^{i}(\bar{z}), & & \tilde{E}^{\alpha}(\bar{z}) \sim: e^{i \alpha . \tilde{\phi}}(\bar{z}): .
\end{array}
$$

Here the symbol $\sim$ indicates the presence of a suitable cocycle factor. Note that $\beta_{n}^{i}=H_{n}^{i}$ and $P^{i}=H_{0}^{i}$. Now given all these, one can explicitly check that in a given formalism (NSR/GS/ Bosonic) the fundamental fields have the same OPE with $T^{S U G}$ and the $T$ defined in that formalism. Also $T^{S U G}$ and all the other energy-momentum tensors have the same central charge $c=4$. This establishes the equivalence of all the energy-momentum tensors. In fact we can write:

$$
T^{N S R}=T^{G S}=T^{B O S} \simeq T^{S U G}
$$

where the symbol $\simeq$ implies that the action of $T^{S U G}$ on any state differs from that of $T^{N S R}, T^{G S}$ or $T^{B O S}$ only by a null state written in terms of the current modes. Here it should be noted that the null states become identically zero when expressed in terms of the fermionic or the bosonic variables, because these realizations are free of null states.

Now we will discuss how type IIA and IIB theories differ as realizations of the same current algebra $\hat{S O}(8)_{k=1}$. To see the difference it is easiest to consider the GS formalism. Let us consider IIB theory in this formalism. Recall that the field content is $S^{a}(z), \tilde{S}^{a}(\bar{z})$ $(a=1,2, . ., 8)$ with integer modes for all fields. Because of the existence of zero modes $S_{0}^{a}$, $\tilde{S}_{0}^{a}$ and their specific anticommutation relations (eqn. (3.6)), the vacuum states (which get annihilated by all the +ve mode oscillators) have the structure [10]

$$
\left(\begin{array}{l}
|\dot{a}\rangle \\
|\mu\rangle
\end{array}\right) \otimes\left(\frac{\widetilde{|\vec{a}\rangle}}{|\mu\rangle}\right) .
$$

(Transformations of these states under $S O(8)$ are given in appendix A.) Since both the left and right sectors have the same structure, we analyze the spectrum built up only on one sector. The spectrum is obtained by acting $\chi_{n}^{ \pm \delta_{i}} \quad(n<0, i=1, . ., 4)$ in all possible ways on the vacuum states, where the modes $\chi_{n}^{ \pm \delta_{i}}$ are defined by taking the same linear 
combinations as in eqns.(3.10). One can show that the $S O(8)$ weight vectors corresponding to the states obtained in this way can be written in the following way:

$$
P=\sum_{i} n_{i}^{\delta} \delta_{i}+e_{1}, \quad n_{i}^{\delta} \in \mathbf{Z}
$$

where $e_{1}$ and $\delta_{i}$ are defined in eqns. (2.4) and (A.16) respectively. It should be noted that $e_{1}$ here, is merely a choice. $P$ takes the same form $(3.22)$ if we replace $e_{1}$ by any weight in $\Pi_{0}^{(e)}$ or $\Pi_{0}^{(\bar{\delta})}$ (see appendix A). Now using the explicit form of the roots (eqn.(2.8)), the weights $\delta_{i}$ (eqn. A.16) ) and $\bar{\delta}_{2}$ (eqn. A.10) ), one can show that

1. When $\sum_{i} n_{i}^{\delta}=$ odd,

$$
P=P^{o d d}=\sum_{i} \bar{n}_{i}^{s} \alpha_{i}^{s}+\bar{\delta}_{2}
$$

where $\bar{n}_{i}^{s} \in \mathbf{Z}$. Furthermore given any set of integer values for $\left\{\bar{n}_{i}^{s}\right\}$ in (3.23), we can find integers $\left\{n_{i}^{\delta}\right\}$ satisfying eqns. (3.22) and (3.23) with $\sum_{i} n_{i}^{\delta}=$ odd.

2. When $\sum_{i} n_{i}^{\delta}=$ even,

$$
P=P^{e v e n}=\sum_{i} n_{i}^{s} \alpha_{i}^{s}+e_{1}
$$

where $n_{i}^{s} \in \mathbf{Z}$. Also given any set of integer values for $\left\{n_{i}^{s}\right\}$ in (3.24), $\left\{n_{i}^{\delta}\right\}$ defined through eqns. (3.22) and (3.24), takes integer values with the condition $\sum_{i} n_{i}^{\delta}=$ even.

These imply that the set of $S O(8)$ weight vectors $\{P\}$ in the spectrum is the union of two disjoint subsets namely $\left\{P^{\text {even }}\right\}$ and $\left\{P^{\text {odd }}\right\}$, and they cover fully the two parts of the weight lattice: $\Lambda_{e}$ and $\Lambda_{\bar{\delta}}$ respectively. Therefore the spectrum is the direct sum of $\Pi^{(e)}$ and $\Pi^{(\bar{\delta})}$, where $\Pi^{(w)}$ is the full irreducible representation built up over the vacuum representation $\Pi_{0}^{(w)}$. Hence the full spectrum ${ }^{[2]}$ of the IIB theory can be expressed in terms of the irreducible components of $\hat{S O}(8)_{k=1}$ in the following way:

$$
\text { type IIB } \quad: \quad\left(\Pi^{(e)} \oplus \Pi^{(\bar{\delta})}\right) \otimes\left(\Pi^{(e)} \oplus \Pi^{(\bar{\delta})}\right) .
$$

In type IIA theory the field content for the left sector is the same as that in type IIB, but for the right sector $\tilde{S}^{a}(\bar{z})$ is replaced by $\tilde{S}^{\dot{a}}(\bar{z})$. Similar analysis shows that the spectrum of IIA theory, in terms of irreducible components of $\hat{S O}(8)_{k=1}$, takes the following form:

$$
\text { type IIA : } \quad\left(\Pi^{(e)} \oplus \Pi^{(\bar{\delta})}\right) \otimes\left(\Pi^{(e)} \oplus \Pi^{(\delta)}\right) .
$$

\footnotetext{
${ }^{12}$ We remind the reader that here we are dealing with the world-sheet fermionic part only.
} 
At this point one notices that $\Pi^{(0)}$ is absent in both the theories. Similar analysis can be done in the NSR formalism. Thus for example in IIB, the NS sector states after GSO projection belong to $\Pi^{(e)}$ and R sector states, after GSO projection, belong to $\Pi^{(\bar{\delta})}$.

Now a final comment about the fermionic realizations: the current algebraic vacuum states $|\mu\rangle,|a\rangle,|\dot{a}\rangle$ appear as representations of various zero-mode oscillators of IIA and IIB theories in different realizations. Thus the latter states are same as the current algebraic vacuum states up to some overall phases. We will take this phase to be 1 in each case.

\section{Ishibashi States and Type IIB D-Branes}

\subsection{Ishibashi States}

Let us consider a two dimensional conformal field theory on the complex plane, both the left and right parts of which realize the same current algebra $\hat{g}$. If $J^{a}(z)$ and $\tilde{J}^{a}(\bar{z})$ are the local currents (in the covariant basis) with modes $J_{n}^{a}$ and $\tilde{J}_{n}^{a}$ respectively, then one considers certain boundary conditions connecting $J^{a}(z)$ and $\tilde{J}^{a}(\bar{z})$ at the boundary $(|z|=|\bar{z}|=1)^{[3}$ which can be written in terms of their modes in the following way:

$$
J_{n}^{a}+\tau\left(\tilde{J}_{-n}^{a}\right)=0
$$

Here $\tau$ is an inner or outer automorphism of $\hat{g}$. An Ishibashi state is a solution of the above boundary condition and is given by [20]:

$$
|w\rangle\rangle=\sum_{N}|N, w\rangle \otimes \mathcal{T} \Theta \mid \widetilde{N, w\rangle}
$$

where $\{|N, w\rangle\}$ is a complete set of orthonormal basis vectors for an unitary irreducible representation of $\hat{g}$ labelled by the highest weight $w$. $\Theta$ is an antiunitary operator on the Hilbert space defined by the following action:

$$
\begin{aligned}
\Theta \tilde{J}_{n}^{a} \Theta^{-1} & =-\tilde{J}_{n}^{a}, \\
\Theta|w\rangle & =|-w\rangle,
\end{aligned}
$$

where $\widetilde{|w\rangle}$ is the highest weight state defined in eqns. (2.12). $\mathcal{T}$ is the Hilbert space operator corresponding to the automorphism $\tau$, and acts only on the right part:

$$
\mathcal{T} \tilde{J}_{n}^{a} \mathcal{T}^{-1}=\tau\left(\tilde{J}_{n}^{a}\right)
$$

One has to properly take into account the action of $\mathcal{T}$ on $|w\rangle$ also. It has been shown in ref. 20] that the fact that $|w\rangle\rangle$ satisfies the boundary condition (4.1) is independent of the choice of the orthonormal basis $\{|N, w\rangle\}$.

\footnotetext{
${ }^{13}$ On the cylinder, $t=0$ is cosidered to be the boundary which under the conformal transformation $z=e^{t} e^{-i \sigma}$, gets mapped on to the unit circle $|z|=|\bar{z}|=1$ on the $z$-plane.
} 


\subsection{Type IIB D-Branes}

We will use the Ishibashi states defined above to derive all the D-brane boundary states in type IIB. There are two points involved - the boundary conditions given by $\tau$ (eqn. (4.1)) or equivalently the Hilbert space operator $\mathcal{T}$ (eqn. (4.4)) and the explicit construction of the basis. From eqn. (4.2) it is clear that after constructing the basis one has to implement the action of $\mathcal{T} \Theta$ on the basis states (only right part). As has been discussed in sec. 1 we will consider $\mathcal{T}$ to be corresponding to the reflection along a set of directions.

Now we will say something about the basis construction. The boundary state does not depend on the specific basis chosen. The basis can be given purely in terms of the current modes or since the current algebra has different realizations, it could be given in terms of the variables of a specific realization. A basis which looks simple in one realization may have very complicated expression in some other realization. We will show that choosing the basis properly in NSR and GS formalisms we can make the expressions for the BPS boundary states look exactly like the already existing solutions for those in the two formalisms. We will also show that the chosen basis in NSR is good enough for expressing also the non-BPS boundary states in that formalism. This however is not true in case of the GS formalism. We will explain why one needs to consider a different basis for expressing the non-BPS branes in GS formalism.

Current Algebraic derivation of BPS results of IIB in NSR and GS formalisms: Let us consider a BPS brane $D p$ for $p=$ odd. We specify its alignment by an ordered set of indices $\left\{\mu_{1}, \mu_{2}, \cdots, \mu_{p+1}\right\}$ with the following chosen ordering: $\mu_{1}<\mu_{2}<\cdots<\mu_{p+1}$. Before we go into the derivation of the corresponding boundary state, we give some definitions and fix some conventions that are needed for the derivation. We define the following matrices [15] corresponding to the above mentioned ordered set of indices:

$$
\begin{aligned}
& M_{\mu \nu}=\left\{\begin{array}{lll}
\delta_{\mu \nu} & \mu \notin\left\{\mu_{i} \mid i=1, \cdots, p+1\right\}, & \nu=1,2, \cdots, 8, \\
-\delta_{\mu \nu} & \mu \in\left\{\mu_{i} \mid i=1, \cdots, p+1\right\}, & \nu=1,2, \cdots, 8,
\end{array}\right. \\
& M_{a b}=\left(\gamma^{\mu_{1}} \bar{\gamma}^{\mu_{2}} \gamma^{\mu_{3}} \bar{\gamma}^{\mu_{4}} \cdots \gamma^{\mu_{p}} \bar{\gamma}^{\mu_{p+1}}\right)_{a b} \text {, } \\
& M_{\dot{a} \dot{b}}=\left(\bar{\gamma}^{\mu_{1}} \gamma^{\mu_{2}} \bar{\gamma}^{\mu_{3}} \gamma^{\mu_{4}} \ldots \bar{\gamma}^{\mu_{p}} \gamma^{\mu_{p+1}}\right)_{\dot{a} \dot{b}} \text {. }
\end{aligned}
$$

Following are some useful properties satisfied by the above matrices [4:

$$
\begin{gathered}
M_{a b} M_{c b}=\delta_{a c}, \quad M_{\dot{a} \dot{b}} M_{\dot{c} \dot{b}}=\delta_{\dot{a} \dot{c}} \\
\gamma_{a \dot{a}}^{\mu}-M_{\dot{a} \dot{b}} M_{a b} M_{\mu \nu} \gamma_{b \dot{b}}^{\nu}=0 .
\end{gathered}
$$

These matrices are respectively the $S O(8)$ vector, spinor and conjugate spinor representations of the reflections along $\mu_{1}, \mu_{2}, \ldots, \mu_{p+1}$. Below we enlist the conventions that we will follow:

\footnotetext{
${ }^{14}$ We could change the definition of $M_{a b}$ and $M_{\dot{a} \dot{b}}$ by a (-) sign without affecting these properties, but this ambiguity does not affect the physical results.
} 
1. The 16 dimensional representaion, (spinor $\oplus$ conjugate spinor) for $\Psi_{0}^{\mu}$, is obtained by applying various $\Psi_{0}^{\mu}$ oscillators on the highest weight state $|\delta\rangle$ which is a spin field acting on the SL $(2, \mathbf{C})$ vacuum. Our convention is to take this spin field to be anticommuting with the right moving fields $\tilde{\Psi}^{\mu}$. Now even number of $\Psi_{0}^{\mu}$ oscilltors act on $|\delta\rangle$ to give spinor representation and odd number to give conjugate spinor representation. Therefore $\tilde{\Psi}_{0}^{\mu}$ goes through $|\dot{a}\rangle$ and picks up a $(-) \operatorname{sign}$ for $|a\rangle$. Due to the similar convention in GS formalism, $\tilde{S}_{0}^{a}$ passes through $|\dot{a}\rangle$ and picks up a $(-)$ sign for $|\mu\rangle$.

2. The GSO rules for the $\mathrm{R}$ sector vacuum states are taken to be:

$$
(-1)^{f}\left(\begin{array}{l}
|a\rangle \\
|\dot{a}\rangle
\end{array}\right)=\left(\begin{array}{c}
-|a\rangle \\
|\dot{a}\rangle
\end{array}\right)
$$

where $f$ is the left moving world-sheet fermion number. Since we are considering IIB, the GSO rules for the right part is also the same.

Let us first derive the NSR BPS states through explicit basis construction. The R-R part of the boundary state corresponds to the irreducible representation $\left(\Pi^{(\bar{\delta})} \otimes \Pi^{(\bar{\delta})}\right)$ (eqn.(3.25)). We choose the following basis (eqn. (4.2)) in $\Pi^{(\bar{\delta})}$ for the left part:

$$
\begin{aligned}
\{|N, \bar{\delta}\rangle\} \equiv\{ & \left\{\prod_{n>0,}\left(\Psi_{-n}^{\mu}\right)^{N_{\mu n}}|\dot{a}\rangle\right]_{\sum N_{\mu n}=\text { even }}, \\
& {\left.\left[\prod_{\substack{n>0, \mu}}\left(\Psi_{-n}^{\mu}\right)^{N_{\mu n}}|a\rangle\right]_{\sum N_{\mu n}=o d d}\right\}, }
\end{aligned}
$$

where $N_{\mu n}=0,1$ and the products are ordered products with some chosen ordering. The states $|a\rangle$ are absent in the representation $\Pi^{(\bar{\delta})}$ and should be understood as being created by applying the $\Psi$ zero-modes on the states $|\dot{a}\rangle$. We may define these in the following way:

$$
|a\rangle=\sqrt{2} \gamma_{a \dot{b}}^{\mu} \Psi_{0}^{\mu}|\dot{b}\rangle
$$

The basis of states on the right part is defined similarly by replacing the left handed oscillators by the right handed ones in eqn. (4.8). The states which appear in the Ishibashi state are:

$$
\{|N, \bar{\delta}\rangle \otimes|\widetilde{N, \bar{\delta}}\rangle\}=\left\{\left|\left\{N_{\nu n}\right\}_{(\text {even })}, \dot{a}\right\rangle, \quad\left|\left\{N_{\nu n}\right\}_{(\text {odd })}, a\right\rangle\right\}
$$

where,

$$
\left|\left\{N_{\mu n}\right\}_{(e v e n)}, \dot{a}\right\rangle \equiv\left[\prod_{\substack{n>0, \mu}}\left(\Psi_{-n}^{\mu}\right)^{N_{\mu n}}|\dot{a}\rangle \otimes \prod_{\substack{n>0, \mu}}\left(\tilde{\Psi}_{-n}^{\mu}\right)^{N_{\mu n}} \widetilde{\dot{a}\rangle}\right]_{\sum_{N_{\mu n}=e v e n}}
$$




$$
\begin{aligned}
& =\left[\prod_{n>0}\left(i \Psi_{-n}^{\mu} \tilde{\Psi}_{-n}^{\mu}\right)^{N_{\mu n}}|\dot{a}\rangle \otimes \widetilde{|\vec{a}\rangle}\right]_{\sum N_{\mu n}=e v e n} \\
\left|\left\{N_{\mu n}\right\}_{(o d d)}, a\right\rangle & \equiv\left[\prod_{n>0,}\left(\Psi_{-n}^{\mu}\right)^{N_{\mu n}}|a\rangle \otimes \prod_{n>0}\left(\tilde{\Psi}_{-n}^{\mu}\right)^{N_{\mu n}}|\widetilde{a}\rangle\right]_{\sum N_{\mu n}=\text { odd }} \\
& \left.=i\left[\prod_{n>0,}\left(i \Psi_{-n}^{\mu} \tilde{\Psi}_{-n}^{\mu}\right)^{N_{\mu n}}|a\rangle \otimes \widetilde{a}\right\rangle\right]_{\sum N_{\mu n}=o d d} .
\end{aligned}
$$

Here we have used the anticommutation rule of $\Psi_{n}^{\mu}$ with $\tilde{\Psi}_{m}^{\nu}$, and the fact that $\tilde{\Psi}_{n}^{\mu}$ passes through $|\dot{a}\rangle$ without picking any $(-)$ sign, and picks up a $(-)$ sign while passing through $|a\rangle$ (see convention (1). Now we have to find the action of $\Theta$ on the states in eqns. (4.11) and (4.12). Using eqns. (4.3), (3.18), (A.9), (A.12) and (A.13) and the fact that $\Theta$ is antilinear, one derives:

$$
\begin{aligned}
\Theta \mid \widetilde{\dot{a}\rangle} & =\widetilde{|\dot{a}\rangle}, \\
\Theta \tilde{\Psi}_{n}^{\mu} \Theta^{-1} & =\tilde{\Psi}_{n}^{\mu} .
\end{aligned}
$$

This with eqns. (4.9), (4.11) and (4.12) says that all the states in (4.10) are invariant under $\Theta$. For the $D p$-brane that we are considering, we have the following action of $\mathcal{T}$ (eqn 4.2):

$$
\begin{gathered}
\mathcal{T} \tilde{\Psi}_{n}^{\mu} \mathcal{T}^{-1}=M_{\mu \nu} \tilde{\Psi}_{n}^{\nu}, \\
\mathcal{T}|\widetilde{a}\rangle=M_{a b}|\widetilde{b}\rangle, \quad \mathcal{T}|\widetilde{\dot{a}}\rangle=M_{\dot{a} \dot{b}}|\widetilde{\dot{b}}\rangle
\end{gathered}
$$

Using eqns. (4.11), (4.12) and (4.14) and the fact that $\Theta$ acts trivially on the states in (4.10) we get:

$$
\begin{array}{r}
\mathcal{T} \Theta \sum_{\left\{N_{\mu n}\right\}, \dot{a}}^{\text {even }}\left|\left\{N_{\mu n}\right\}_{(\text {even })}, \dot{a}\right\rangle=\sum_{\left\{N_{\mu n}\right\}, \dot{a}, \dot{b}}^{\text {even }} \prod_{n>0,}\left(i \Psi_{-n}^{\mu}\left(\sum_{\nu} M_{\mu \nu} \tilde{\Psi}_{-n}^{\nu}\right)\right)^{N_{\mu n}} \\
M_{\dot{a} \dot{b}}|\dot{a}\rangle \otimes \mid \widetilde{\dot{b}\rangle}, \\
\mathcal{T} \Theta \sum_{\left\{N_{\mu n}\right\}, a}^{\text {odd }}\left|\left\{N_{\mu n}\right\}_{(\text {odd })}, a\right\rangle=i \sum_{\left\{N_{\mu n}\right\}, a, b}^{\text {odd }} \prod_{n>0,}\left(i \Psi_{-n}^{\mu}\left(\sum_{\nu} M_{\mu \nu} \tilde{\Psi}_{-n}^{\nu}\right)\right)^{N_{\mu n}} \\
M_{a b}|a\rangle \otimes|\widetilde{b}\rangle
\end{array}
$$


where $\sum_{\left\{N_{\mu n}\right\}}^{\text {even }}$ and $\sum_{\left\{N_{\mu n}\right\}}^{\text {odd }}$ imply sum over the set $\left\{N_{\mu n}\right\}$ of numbers $N_{\mu n}=0,1$ with $\sum_{n>0} N_{\mu n}=$ even and odd respectively. Then using eqns. (4.2), (4.10) and (4.15) it is straightforward to show that the Ishibashi state $|p, \bar{\delta}\rangle\rangle$ corresponding to the specific $D$-brane we are considering is given by:

$$
|p, \bar{\delta}\rangle\rangle=|p, R R\rangle_{N S R}
$$

where

$$
\begin{aligned}
|p, R R\rangle_{N S R} & =\frac{1}{2}[|p, R R,+\rangle+|p, R R,-\rangle] \\
|p, R R, \pm\rangle & =\exp \left[ \pm i \sum_{n>0} \Psi_{-n}^{\mu} M_{\mu \nu} \tilde{\Psi}_{-n}^{\nu}\right]|p, R R, \pm\rangle_{0} \\
|p, R R, \pm\rangle_{0} & \left.=M_{\dot{a} \dot{b}}|\dot{a}\rangle \otimes \widetilde{\dot{b}\rangle} \pm i M_{a b}|a\rangle \otimes \widetilde{b}\right\rangle
\end{aligned}
$$

According to the convention adopted for the GSO rules, $|p, R R\rangle_{N S R}$ is GSO invariant. One can show that the R-R part of the actual $D p$ brane boundary state written in the NSR formalism takes the form $\mathcal{A}|p, R R\rangle_{N S R}$, where the operator $\mathcal{A}$ does not involve any fermionic oscillator.

For the NS-NS part of the boundary state one starts with the irreducible representation $\left(\Pi^{(e)} \otimes \Pi^{(e)}\right)$ (eqn.(3.25)) and proceeds in the same way. For constructing $\left.|p, e\rangle\right\rangle$ we choose the basis states to be,

$$
\{|N, e\rangle\} \equiv\left\{\left[\prod_{\substack{r>0, \mu}}\left(\Psi_{-r}^{\mu}\right)^{N_{\mu r}}|0\rangle\right]_{\sum N_{\mu r}=o d d}\right\} .
$$

The final result is

$$
|p, e\rangle\rangle=|p, N S N S\rangle_{N S R},
$$

where

$$
\begin{aligned}
|p, N S N S\rangle_{N S R} & =\frac{1}{2}[|p, N S N S,+\rangle-|p, N S N S,-\rangle] \\
|p, N S N S, \pm\rangle & =\exp \left[ \pm i \sum_{r>0} \Psi_{-r}^{\mu} M_{\mu \nu} \tilde{\Psi}_{-r}^{\nu}\right]|0\rangle \otimes \widetilde{0\rangle}
\end{aligned}
$$

Again one can show that the NS-NS part of the actual $D p$ brane boundary state written in the NSR formalism takes the form $\mathcal{A}|p, N S N S\rangle_{N S R}$, with the same operator $\mathcal{A}$ appearing in the R-R part. Therefore $|p, \eta\rangle \equiv|p, e\rangle\rangle+i \eta|p, \bar{\delta}\rangle\rangle$ reproduces the known expression 
for the $D p(\bar{D} p)$-brane boundary state for $\eta=1(\eta=-1)$ in the sense that the complete $D p$-brane boundary state takes the form $\mathcal{A}|p, \eta\rangle$.

For deriving the BPS boundary state in GS formalism we take the following basis:

$$
\begin{gathered}
\{|N, e\rangle\} \equiv\left\{\left[\prod_{a, n>0}\left(S_{-n}^{a}\right)^{N_{a n}}|\mu\rangle\right]_{\sum N_{a n}=\text { even }},\right. \\
\left.\left\{\prod_{a, n>0}\left(S_{-n}^{a}\right)^{N_{a n}}|\dot{a}\rangle\right]_{\sum N_{a n}=o d d}\right\}, \\
\{|N, \bar{\delta}\rangle\} \equiv\left\{\left[\prod_{a, n>0}\left(S_{-n}^{a}\right)^{N_{a n}}|\dot{a}\rangle\right]_{\sum N_{a n}=\text { even }},\right. \\
\left.\left[\prod_{a, n>0}\left(S_{-n}^{a}\right)^{N_{a n}}|\mu\rangle\right]_{\sum N_{a n}=o d d}\right\}
\end{gathered}
$$

and similarly for the right part. Then proceeding in the same way as described above one can show

$$
\begin{aligned}
|p, \eta\rangle & =\exp \left[-i \eta \sum_{n>0} S_{-n}^{a} M_{a b} \tilde{S}_{-n}^{b}\right]|p, \eta\rangle_{0} \\
|p, \eta\rangle_{0} & =M_{\mu \nu}|\mu\rangle \otimes \widetilde{|\nu\rangle}+i \eta M_{\dot{a} \dot{b}}|\dot{a}\rangle \otimes \widetilde{|\dot{b}\rangle} .
\end{aligned}
$$

The above form of $|p, \eta\rangle$ agrees with the known expression [15] in GS formalism[.

\section{Non-BPS boundary states of IIB in NSR formalism and problem with GS formalism:}

Let us consider a non-BPS brane $D p$ ( $p$ even) in IIB with the alignment given by the ordered set of indices $\left\{\mu_{1}, \mu_{2}, \cdots, \mu_{p+1}\right\}_{\mu_{1}<\mu_{2}<\cdots,<\mu_{p+1}}$. The vector, spinor and conjugate spinor representations of the parity transformation along all of these directions are given respectively by:

$$
\begin{aligned}
\bar{M}_{\mu \nu} & =\left\{\begin{array}{ll}
\delta_{\mu \nu} & \mu \notin\left\{\mu_{i} \mid i=1, \cdots, p+1\right\}, \nu=1,2, \cdots, 8, \\
-\delta_{\mu \nu} & \mu \in\left\{\mu_{i} \mid i=1, \cdots, p+1\right\}, \nu=1,2, \cdots, 8,
\end{array},\right. \\
\bar{M}_{a \dot{b}} & =\left(\gamma^{\mu_{1}} \bar{\gamma}^{\mu_{2}} \gamma^{\mu_{3}} \bar{\gamma}^{\mu_{4}} \ldots \gamma^{\mu_{p-1}} \bar{\gamma}^{\mu_{p}} \gamma^{\mu_{p+1}}\right)_{a \dot{b}} \\
\bar{M}_{\dot{a} b} & =\left(\bar{\gamma}^{\mu_{1}} \gamma^{\mu_{2}} \bar{\gamma}^{\mu_{3}} \gamma^{\mu_{4}} \ldots \bar{\gamma}^{\mu_{p-1}} \gamma^{\mu_{p}} \bar{\gamma}^{\mu_{p+1}}\right)_{\dot{a} b} .
\end{aligned}
$$

\footnotetext{
${ }^{15}$ One can again show that $\Theta$ acts trivially on all the right moving basis states. The matrices $M_{\mu \nu}$, $M_{a b}$ and $M_{\dot{a} \dot{b}}$ get introduced in the same way, as we saw in the NSR case, through the action of $\mathcal{T}$.

${ }^{16}$ In the sense that the complete boundary state is $\mathcal{A}|p, \eta\rangle$.
} 
The properties corresponding to that of eqns. (4.6) satisfied by the $\bar{M}$ matrices $\square$ are,

$$
\begin{gathered}
\bar{M}_{a \dot{b}} \bar{M}_{c \dot{b}}=\delta_{a c}, \quad \bar{M}_{\dot{a} b} \bar{M}_{\dot{c} b}=\delta_{\dot{a} \dot{c}} \\
\bar{\gamma}_{\dot{a} a}^{\mu}+\bar{M}_{\dot{a} b} \bar{M}_{a \dot{b}} \bar{M}_{\mu \nu} \bar{\gamma}_{\dot{b} b}^{\nu}=0 .
\end{gathered}
$$

We will discuss the current algebraic derivation of the corresponding boundary state in NSR formalism first. Let us first notice that the Ishibashi state $|p, \bar{\delta}\rangle\rangle$ corresponding to the alignment described above is not realized in IIB. This is because the states belonging to the conjugate spinor representation labelled by the highest weight $\bar{\delta}$ go over to the states in the spinor representation labelled by the highest weight $\delta$ under the action of $\mathcal{T}$ correponding to this non-BPS boundary condition $\$$. Therefore we have:

$$
\mathcal{T}: \quad \Pi^{(\bar{\delta})} \otimes \Pi^{(\bar{\delta})} \rightarrow \Pi^{(\bar{\delta})} \otimes \Pi^{(\delta)} .
$$

But the states in $\left(\Pi^{(\bar{\delta})} \otimes \Pi^{(\delta)}\right)$ are not present in IIB. The Ishibashi state $\left.|p, e\rangle\right\rangle$ does get realized in IIB (see footnote 18) and can be obtained by starting with the same basis (eqn. 4.18) used in the BPS case. Using the following action of $\mathcal{T}$ :

$$
\begin{aligned}
\mathcal{T} \mid \widetilde{0\rangle} & =\widetilde{0\rangle} \\
\mathcal{T} \tilde{\Psi}_{n}^{\mu} \mathcal{T}^{-1} & =\bar{M}_{\mu \nu} \tilde{\Psi}_{n}^{\nu} .
\end{aligned}
$$

one gets,

$$
\begin{aligned}
|p, e\rangle\rangle & =\frac{1}{2}[|p, N S N S,+\rangle-|p, N S N S,-\rangle] \\
|p, N S N S, \pm\rangle & =\exp \left[ \pm i \sum_{r>0} \Psi_{-r}^{\mu} \bar{M}_{\mu \nu} \tilde{\Psi}_{-r}^{\nu}\right]|0\rangle \otimes \mid \widetilde{0\rangle}
\end{aligned}
$$

As in the BPS case, the state $|p, e\rangle\rangle$ agrees with the known expression for the nonBPS boundary state in the sense that the actual non-BPS boundary state has the form $\overline{\mathcal{A}}|p, e\rangle\rangle$, where $\overline{\mathcal{A}}$ is an operator which does not contain the fermionic oscillators.

This procedure does not go through so smoothly in the GS formalism. Here one is supposed to start with the basis (4.21) and apply $\mathcal{T} \Theta$ on the right part. One would naively think that $\mathcal{T}$ has the following action:

$$
\begin{aligned}
\mathcal{T} \widetilde{|\mu\rangle} & =\bar{M}_{\mu \nu} \widetilde{|\nu\rangle}, \\
\mathcal{T} \widetilde{\dot{a}\rangle} & =\bar{M}_{\dot{a} b} \widetilde{|b\rangle}, \\
\mathcal{T} \tilde{S}_{n}^{a} \mathcal{T}^{-1} & =\bar{M}_{a \dot{b}} \tilde{S}_{n}^{\dot{b}} .
\end{aligned}
$$

\footnotetext{
${ }^{17}$ As before, the matrices $\bar{M}_{a \dot{b}}$ and $\bar{M}_{\dot{a} b}$ could be defined with a factor of (-) sign without affecting these properties. But again this ambiguity is not physically relevant.

${ }^{18} \mathrm{~A}$ reflection along odd number of directions corresponds to an outer automorphism of the $\hat{S O}(8)_{k}$ current algebra which interchanges the spinor and conjugate spinor representations but maps the vector representation to itself.
} 
But notice that the states $\widetilde{|b\rangle}$ and the modes $\tilde{S}_{n}^{\dot{b}}$ are not defined in IIB. Although $\mathcal{T}$ does not have well defined action on the above objects in IIB, it does have well defined action on the states in (4.21). This is because, each of these states is either a direct product of even number of spinor representations and the vector representation or odd number of spinor representations and the conjugate spinor representation. Therefore all of them tranform tensorially under $S O(8)$ and pick up some factor of $(-)$ signs under $\mathcal{T}$ (since it is a reflection along certain directions). So to find the action of $\mathcal{T}$ on these states one needs to perform the tensorial decomposition of them. This, unfortunately is a difficult task.

Thus we see that the problem arises because of the specific variables that we are working with or equivalently the specific choice of basis that we have made. It seems that it is best to come out of the language of this specific realization and adopt that of the curent algebra, which is universal, and then reexpress these basis states in the GS formalism.

\section{Basis Construction and the Non-BPS Boundary States}

\subsection{Basis Construction}

An irreducible representation $\Pi^{(w)}$ is obtained by applying $H_{-n}^{i}, E_{-n}^{ \pm \alpha}$ and $E_{0}^{-\alpha}$ on the corresponding highest weight state $|w\rangle$ in all possible ways, where $i=1, . ., 4, n>0$ and $\alpha \in \Delta^{+}, \Delta^{+}$being the set of all positive roots. The Hilbert space contains null states, which one has to put to zero. Using eqns (2.5), (2.6), (2.7) and the fact that any $\alpha \in \Delta$ (set of all roots) can be written as a linear combination of the simple roots $\alpha_{i}^{s}$ 's with integer coefficients, one can argue that it is sufficient to use $H_{-n}^{i}(n>0)$ and $E_{-n}^{ \pm \alpha_{i}^{s}}(n \geq 0)$ to build up the spectrum. But since $E_{-n}^{ \pm \alpha_{i}^{s}}$ 's $(n \geq 0)$ do not commute, using these is inconvenient. We solve this problem by treating the vacuum states (which are finite in number $)$ in a special way and using the commuting modes $E_{-n}^{ \pm \alpha_{i}}$ 's $(n>0, i=1, \ldots, 4)$ with $\alpha_{i}$ 's defined in eqns. (2.8), instead of $E_{-n}^{ \pm \alpha_{i}^{s}}$ 's. Our basis states would look exactly similar to those one would have constructed in the bosonic fomalism. In this formalism, a basis state is given certain compact momentum $(P)$ and raised to some energy by applying the negative mode oscillators $\beta_{-n}^{i}$ 's on the bosonic vacuum. We will use the $E_{-n}^{\alpha_{i}}$ oscillators to create the compact momentum (i.e to fix the position on the weight lattice) and use $H_{-n}^{i}\left(=\beta_{-n}^{i}\right)^{\prime}$ 's to lift it to any desired energy. The construction will be free of null states.

Since these basis states will be much like the bosonic states, it is useful to consider the bosonic expression for the zero-mode of the energy-momentum tensor:

$$
L_{0}^{B O S}=\frac{1}{2} P^{2}+N^{H}
$$


where $P^{2}=\sum_{i} P^{i} P^{i}, P^{i}=H_{0}^{i}$ and $N^{H}=\sum_{i} \sum_{n>o} H_{-n}^{i} H_{n}^{i}$. We will call a state a bosonic vacuum state minimum value for the bosonic vacuum state. While constructing the basis we will use the fact that any state in the free module generated by $J_{-n}^{\mu \nu}$ 's $(n \geq 0)$ having $L_{0}^{B O S}$ eigenvalue less than $\frac{1}{2} P^{2}$ eigenvalue, should be null. The same argument may be applied to show:

$$
H_{n}^{i}\left|P, N^{H}=0\right\rangle=0, \quad \forall n>0,
$$

where $\left|P, N^{H}=0\right\rangle$ is a bosonic vacuum state.

Let us consider $\Pi^{(e)}$ first. The current algebra vacuum states, annihilated by $J_{n}^{\mu \nu}$, $(\forall \mu, \nu$ and $\forall n>0)$ are $\left| \pm e_{i}\right\rangle$ (eqn. (A.4)), where the states are labelled by their $P$ eigenvalue (see eqn. (A.5)). If we adopt a new notation for these states, namely $\left|l_{1}, l_{2}, l_{3}, l_{4}\right\rangle$ with $P=\sum_{i} l_{i} \alpha_{i}$, then we can write,

$$
\begin{aligned}
& \left| \pm e_{1}\right\rangle=\left| \pm \frac{1}{2}, \pm \frac{1}{2}, 0,0\right\rangle, \quad\left| \pm e_{2}\right\rangle=\left| \pm \frac{1}{2}, \mp \frac{1}{2}, 0,0\right\rangle \\
& \left| \pm e_{3}\right\rangle=\left|0,0, \pm \frac{1}{2}, \pm \frac{1}{2}\right\rangle, \quad\left| \pm e_{4}\right\rangle=\left|0,0, \pm \frac{1}{2}, \mp \frac{1}{2}\right\rangle .
\end{aligned}
$$

Now the allowed momenta in $\Pi^{(e)}$ take value in $\Lambda_{e}$. We define a set $\mathcal{K}^{(e)}$ of 4 -vectors $\vec{s} \equiv\left(s_{1}, s_{2}, s_{3}, s_{4}\right)$, such that each $s_{i}(i=1, . ., 4)$ is either integer or half-odd-integer and,

$$
\begin{array}{rr}
\text { either } & s_{1}, s_{2} \in \mathbf{Z}+\frac{1}{2}, \quad s_{3}, s_{4} \in \mathbf{Z}, \\
\text { or } & s_{1}, s_{2} \in \mathbf{Z}, \quad s_{3}, s_{4} \in \mathbf{Z}+\frac{1}{2} .
\end{array}
$$

Then one can show that if $P=\sum_{i} s_{i} \alpha_{i} \in \Lambda_{e}$, then $\vec{s} \in \mathcal{K}^{(e)}$ and vice versa. Notice that the $\left| \pm e_{i}\right\rangle$ 's are examples of this. The bosonic vacuum state with momentum $P=\sum_{i} s_{i} \alpha_{i} \in \Lambda_{e}$ can be expressed as,

$$
\left|\vec{s} \in \mathcal{K}^{(e)}\right\rangle=\prod_{i} \mathcal{E}^{\alpha_{i}}\left(s_{i}\right)|V\rangle
$$

where the state $|V\rangle$ and the operators $\mathcal{E}^{\alpha_{i}}\left(s_{i}\right)$ are defined as follows:

$$
|V\rangle \equiv \begin{cases}\left| \pm e_{1}\right\rangle & s_{1}, s_{2} \in \mathbf{Z}_{\frac{1}{2}}^{ \pm}, \\ \left| \pm e_{2}\right\rangle & s_{1} \in \mathbf{Z}_{\frac{1}{2}}^{ \pm}, s_{2} \in \mathbf{Z}_{\frac{1}{2}}^{\mp} \\ \left| \pm e_{3}\right\rangle & s_{3}, s_{4} \in \mathbf{Z}_{\frac{1}{2}}^{ \pm}, \\ \left| \pm e_{4}\right\rangle & s_{3} \in \mathbf{Z}_{\frac{1}{2}}^{ \pm}, s_{4} \in \mathbf{Z}_{\frac{1}{2}}^{\mp} .\end{cases}
$$

\footnotetext{
${ }^{19}$ Bosonic vacuum states should not be confused with the vacuum representation of the current algebra.
} 


$$
\mathcal{E}^{\alpha}(s) \equiv \begin{cases}E_{-2|s|+1}^{ \pm \alpha} E_{-2|s|+3}^{ \pm \alpha} \cdots E_{-2}^{ \pm \alpha} & s \in \mathbf{Z}_{\frac{1}{2}}^{ \pm} \\ E_{-2|s|+1}^{ \pm \alpha} E_{-2|s|+3}^{ \pm \alpha} \cdots E_{-1}^{ \pm \alpha} & s \in \mathbf{Z}^{ \pm} \\ 1 & s=0, \quad \pm \frac{1}{2}\end{cases}
$$

where $\mathbf{Z}^{ \pm}$are respectively the sets of positive and negative integers and $\mathbf{Z}_{\frac{1}{2}}^{ \pm}$are respectively the sets of positive and negative half-odd-integers. As all $E^{\alpha_{i}}$ 's are commuting, the ordering of the operators $\mathcal{E}^{\alpha_{i}}\left(s_{i}\right)$ in the product in eqn. (5.5) is not important.

The fact that the state $|\vec{s}\rangle$ defined above, is a bosonic vacuum state can be proved as follows: using eqn. (2.6) for $m=0$ and eqn. (A.5), it can be shown that,

$$
P|\vec{s}\rangle=\vec{s}|\vec{s}\rangle \text {. }
$$

From the Sugawara's construction of the energy-momentum tensor (eqns. (2.10), (2.11)), the following commutation relation can be established:

$$
\left[L_{0}^{S U G}, J_{n}^{\mu \nu}\right]=-n J_{n}^{\mu \nu}, \quad \forall n \in \mathbf{Z} .
$$

The $L_{0}^{S U G}$ eigenvalues of the vacuum states can also be found:

$$
L_{0}^{S U G}\left| \pm e_{i}\right\rangle=\frac{1}{2} P^{2}\left| \pm e_{i}\right\rangle=\frac{1}{2}\left| \pm e_{i}\right\rangle
$$

Then using eqns. (5.9) and (5.10), one can show that,

$$
L_{0}^{S U G}|\vec{s}\rangle=\frac{1}{2} P^{2}|\vec{s}\rangle=\left(\sum_{i} s_{i}^{2}\right)|\vec{s}\rangle .
$$

Hemce $|\vec{s}\rangle$ is a bosonic vacuum state.

In Appendix $\mathrm{C}$ we show that the norm of a bosonic vacuum state constructed above is 1. The orthogonality of the basis states can be shown as follows: for a given momentum eigenvalue corresponding to a point on the lattice $\Lambda_{e}$, there is only one linearly independent state which qualifies as a bosonic vacuum state (this fact is manifest in the bosonic formalism). Now since $H_{0}^{i}=P^{i}$ is a hermitian operator, states with different momentum eigenvalues must be orthogonal.

Finally the full set of orthonormal basis in $\Pi^{(e)}$ is given by $\left\{\left|\vec{s} \in \mathcal{K}^{(e)},\left\{N_{i n}\right\}\right\rangle\right\}$,

$$
\left|\vec{s} \in \mathcal{K}^{(e)}, \quad\left\{N_{i n}\right\}\right\rangle \equiv \prod_{\substack{n>0, i}} \frac{\left(\frac{1}{\sqrt{n}} H_{-n}^{i}\right)^{N_{i n}}}{\sqrt{N_{i n} !}}\left|\vec{s} \in \mathcal{K}^{(e)}\right\rangle
$$


with $N_{\text {in }}=0,1,2, \cdots \forall i, \forall n>0$. The following orthonormality condition of this complete set of basis states can be checked by using eqn. (2.5) and the orthonormality of the bosonic vacuum states:

$$
\left\langle\vec{s}^{\prime} \in \mathcal{K}^{(e)},\left\{N_{i n}^{\prime}\right\} \mid \vec{s} \in \mathcal{K}^{(e)},\left\{N_{i n}\right\}\right\rangle=\delta_{\vec{s}^{\prime}, \vec{s}} \delta_{\left\{N_{i n}^{\prime}\right\},\left\{N_{i n}\right\}}
$$

The right moving states are defined, as before, by replacing the left handed oscillators by the right handed ones. The same procedure can be followed for $\Pi^{(\bar{\delta})}$ and $\Pi^{(\delta)}$. But we do not need the explicit expressions for these two, as we are interested in writing down the non-BPS states which involve only $\Pi^{(e)}$.

\subsection{Non-BPS Boundary States}

We have seen in subsec. 4.2 that the actual non-BPS boundary state takes the form $\overline{\mathcal{A}}|p, e\rangle\rangle$, where $\overline{\mathcal{A}}$ is an operator which does not involve fermionic oscillators and hence takes the same form in both NSR and GS formalisms. Therefore it is sufficient to consider only the Ishibashi state corresponding to the highest weight $w=e$ for a given non-BPS alignment and try to express that in terms of the current modes. Let us start with the following Ishibashi state:

$$
|\mathcal{N}, e\rangle\rangle=\sum_{\vec{s} \in \mathcal{K}^{(e),\left\{N_{i n}\right\}}}\left|\vec{s},\left\{N_{i n}\right\}\right\rangle \otimes \mathcal{T}_{\mathcal{N}} \Theta\left|\vec{s},\left\{\widetilde{\{N}_{i n}\right\}\right\rangle
$$

where $\mathcal{T}_{\mathcal{N}}$ is the Hilbert space operator (acting only on the right part) corresponding to the reflection along the following set of ordered directions:

$$
\mathcal{N}=\left\{\mu_{1}, \mu_{2}, \cdots, \mu_{p+1}\right\}_{\mu_{1}<\mu_{2}<\cdots<\mu_{p+1}} .
$$

In the previous subsection the basis states have been written purely in terms of the currents. Once we get the action of $\mathcal{T}_{\mathcal{N}} \Theta$ on these and finally express everything in terms of the current modes, the above Ishibashi state can be translated into the language of any of the three realizations considered in sec. 3. Since the above state involves only the NS-NS sector exitations, this is realized both in IIA and IIB. When realized in IIA with $p=$ odd, this state gives a non-BPS state in IIA. Similarly in IIB, this gives a non-BPS state for $p=$ even.

Let us now analyze the action of $\Theta$ on the basis states. Using eqns. (4.3), (2.2), (2.3), (A.6) and (A.7) and the fact that $\Theta$ is antilinear, we get the following:

$$
\begin{aligned}
\Theta \tilde{H}_{n}^{i} \Theta^{-1} & =-\tilde{H}_{n}^{i}, & & \forall i, \forall n>0, \\
\Theta \tilde{E}_{n}^{\alpha_{i}} \Theta^{-1} & =-\tilde{E}_{n}^{-\alpha_{i}}, & & \forall i, \forall n>0, \\
\Theta\left| \pm e_{i}\right\rangle & =\left|\mp e_{i}\right\rangle, & & \forall i .
\end{aligned}
$$


Now applying the second and third eqns. of (5.16) and eqns. (5.5), (5.6), (5.7) one can show:

$$
\Theta \widetilde{\vec{s}\rangle}=(-1) \sum_{i}\left|s_{i}\right|-1 \widetilde{-\vec{s}\rangle} \equiv|\overline{-\vec{s}}\rangle
$$

where $\vec{s} \in \mathcal{K}^{(e)}$. Then finally using the eqns. (5.14), (5.12), (5.17) and the first eqn. of (5.16) one can show:

$$
|\mathcal{N}, e\rangle\rangle=\mathcal{T}_{\mathcal{N}} \exp \left[-\sum_{\substack{n>0 \\ i}} \frac{1}{n} H_{-n}^{i} \tilde{H}_{-n}^{i}\right] \sum_{\vec{s} \in \mathcal{K}^{(e)}}|\vec{s}\rangle \otimes|\overline{-\vec{s}}\rangle .
$$

Now let us define a 4 -vector $\vec{n}(\vec{s})=\left(n_{1}(\vec{s}), n_{2}(\vec{s}), n_{3}(\vec{s}), n_{4}(\vec{s})\right)$ by,

$$
\sum_{i} n_{i}(\vec{s}) e_{i}=\sum_{i} s_{i} \alpha_{i}
$$

Then one can show that $\forall \vec{s} \in \mathcal{K}^{(e)}, \exists$ a unique $\vec{n}(\vec{s})$ such that $n_{i}(\vec{s}) \in \mathbf{Z}$, and $\sum_{i} n_{i}(\vec{s})=$ odd and vice versa. Therefore there is a one-to-one correspondence between the vectors $\vec{s}$ and $\vec{n}$. We will adopt the following notation for the Ishibashi state (5.18) in future:

$$
|\mathcal{N}, e\rangle\rangle=\mathcal{T}_{\mathcal{N}} \exp \left[-\sum_{\substack{n>0 \\ i}} \frac{1}{n} H_{-n}^{i} \tilde{H}_{-n}^{i}\right] \sum_{\vec{n}}^{o d d}|\vec{s}(\vec{n})\rangle \otimes|\overrightarrow{-\vec{s}(\vec{n})}\rangle
$$

where $\sum_{\vec{n}}^{\text {odd }}$ represents sum over integer $n_{i}$ 's with $\sum_{i} n_{i}=$ odd and $\vec{s}(\vec{n})$ is the inverse function of $\vec{n}(\vec{s})$. For $\mathcal{N}=\{$ null $\}$, i.e., $\mathcal{T}_{\mathcal{N}}=$ identity, this state, expressed in terms of IIA variables, gives the non-BPS D-instanton in IIA (also this same state, expressed in terms of the IIB variables, gives the NS-NS part of the BPS D-instanton boundary state in IIB). Let us now consider a nontrivial case where $\mathcal{T}_{\mathcal{N}}$ is not identity.

Reflection along the direction $\mu=2$ : In this case $\mathcal{N}=\{2\}$. Equation (4.4) reads:

$$
\mathcal{T}_{\{2\}} \tilde{J}_{n}^{\mu \nu} \mathcal{T}_{\{2\}}^{-1}= \begin{cases}\tilde{J}_{n}^{\mu \nu}, & \mu \neq 2, \quad \nu \neq 2, \\ -\tilde{J}_{n}^{\mu \nu}, & \text { otherwise }\end{cases}
$$

Then using eqns. (2.2) and (2.3) one shows,

$$
\begin{gathered}
\mathcal{T}_{\{2\}} \tilde{H}_{n}^{i} \mathcal{T}_{\{2\}}^{-1}= \begin{cases}-\tilde{H}_{n}^{i}, & i=1, \\
\tilde{H}_{n}^{i}, & i \neq 1 .\end{cases} \\
\mathcal{T}_{\{2\}} \tilde{E}_{n}^{ \pm \alpha_{1}} \mathcal{T}_{\{2\}}^{-1}=\tilde{E}_{n}^{\mp \alpha_{2}}, \quad \mathcal{T}_{\{2\}} \tilde{E}_{n}^{ \pm \alpha_{2}} \mathcal{T}_{\{2\}}^{-1}=\tilde{E}_{n}^{\mp \alpha_{1}}, \\
\mathcal{T}_{\{2\}} \tilde{E}_{n}^{ \pm \alpha_{3}} \mathcal{T}_{\{2\}}^{-1}=\tilde{E}_{n}^{ \pm \alpha_{3}}, \quad \mathcal{T}_{\{2\}} \tilde{E}_{n}^{ \pm \alpha_{4}} \mathcal{T}_{\{2\}}^{-1}=\tilde{E}_{n}^{ \pm \alpha_{4}} .
\end{gathered}
$$


Action of $\mathcal{T}_{\{2\}}$ on the current algebra vacuum states in the covariant basis is as follows:

$$
\mathcal{T}_{\{2\}} \widetilde{|\mu\rangle}=\left\{\begin{aligned}
-\widetilde{|\mu\rangle}, & \mu=2, \\
\widetilde{|\mu\rangle}, & \mu \neq 2 .
\end{aligned}\right.
$$

Using this and eqn. (A.4) one can show:

$$
\begin{aligned}
& \mathcal{T}_{\{2\}}\left|\widetilde{ \pm e_{1}}\right\rangle=\left|\widetilde{\mp e_{1}}\right\rangle, \\
& \mathcal{T}_{\{2\}}\left|\widetilde{ \pm e_{i}}\right\rangle=\left|\widetilde{ \pm e_{i}}\right\rangle, \quad i \neq 1 .
\end{aligned}
$$

One can check that eqns. (5.25) are consistent with the transformations (5.23) and the eqns. (A.6), (A.7). Now using the transformations (5.23) and (5.25), one can show that,

$$
\left.\mathcal{T}_{\{2\}}|\vec{s}(\vec{n})\rangle \otimes|\overline{-\vec{s}(\vec{n})}\rangle=|\vec{s}(\vec{n})\rangle \otimes \overline{-\vec{s}\left(D_{\{2\}} \vec{n}\right)}\right\rangle
$$

where $D_{\{2\}}=\operatorname{diag}(-1,1,1,1)$. Finally applying eqns. (5.20), (5.22) and (5.26) one gets,

$$
\begin{aligned}
|\{2\}, e\rangle\rangle & =\mathcal{T}_{\{2\}} \exp \left[-\sum_{\substack{n>0 \\
i}} \frac{1}{n} H_{-n}^{i} \tilde{H}_{-n}^{i}\right] \sum_{\vec{n}}^{o d d}|\vec{s}(\vec{n})\rangle \otimes|\overrightarrow{-\vec{s}(\vec{n})}\rangle \\
& =\exp \left[-\sum_{n>0} \frac{1}{n} H_{-n}^{T} D_{\{2\}} \tilde{H}_{-n}\right] \sum_{\vec{n}}^{o d d}|\vec{s}(\vec{n})\rangle \otimes \mid \overrightarrow{\left.-\vec{s}\left(D_{\{2\}} \vec{n}\right)\right\rangle} .
\end{aligned}
$$

We will give explicit expressions like eqn. (5.27) for the Ishibashi states for some special alignments first. We define these special alignments as follows:

$$
\begin{aligned}
& \mathcal{N}_{1}^{A}=\{\text { null }\}, \quad \mathcal{N}_{2}^{A}=\{2,4\}, \quad \mathcal{N}_{3}^{A}=\{2,6\}, \quad \mathcal{N}_{4}^{A}=\{2,8\}, \\
& \mathcal{N}_{5}^{A}=\{4,6\}, \quad \mathcal{N}_{6}^{A}=\{4,8\}, \quad \mathcal{N}_{7}^{A}=\{6,8\}, \quad \mathcal{N}_{8}^{A}=\{2,4,6,8\} \text {. } \\
& \mathcal{N}_{1}^{B}=\{2\}, \quad \mathcal{N}_{2}^{B}=\{4\}, \quad \mathcal{N}_{3}^{B}=\{6\}, \quad \mathcal{N}_{4}^{B}=\{8\}, \\
& \mathcal{N}_{5}^{B}=\{2,4,6\}, \quad \mathcal{N}_{6}^{B}=\{2,4,8\}, \quad \mathcal{N}_{7}^{B}=\{2,6,8\}, \quad \mathcal{N}_{8}^{B}=\{4,6,8\} \text {. }
\end{aligned}
$$

Every special alignment $\mathcal{N}_{\rho}^{A}(\rho=1,2, \ldots, 8)$ corresponds to some non-BPS brane in IIA. Similarly every $\mathcal{N}_{\rho}^{B}$ corresponds to some non-BPS brane in IIB. To get the Ishibashi state for any of these special alignments one follows the same procedure as that we have just described for $\mathcal{N}_{1}^{B}$ (eqn. (5.27)). We give the results below:

$$
\begin{aligned}
& \left.\left|\mathcal{N}_{\rho}^{A}, e\right\rangle\right\rangle=\exp \left[-\sum_{n>0} \frac{1}{n} H_{-n}^{T} D_{\rho}^{A} \tilde{H}_{-n}\right] \sum_{\vec{n}}^{\text {odd }}|\vec{s}(\vec{n})\rangle \otimes\left|\overline{-\vec{s}\left(D_{\rho}^{A} \vec{n}\right)}\right\rangle \\
& \left.\left|\mathcal{N}_{\rho}^{B}, e\right\rangle\right\rangle=\exp \left[-\sum_{n>0} \frac{1}{n} H_{-n}^{T} D_{\rho}^{B} \tilde{H}_{-n}\right] \sum_{\vec{n}}^{\text {odd }}|\vec{s}(\vec{n})\rangle \otimes\left|\overline{-\vec{s}\left(D_{\rho}^{B} \vec{n}\right)}\right\rangle
\end{aligned}
$$




\begin{tabular}{|c|c|c|}
\hline \hline$\rho$ & $D_{\rho}^{A}$ & $D_{\rho}^{B}$ \\
\hline \hline 1 & $\mathbf{1}_{4}$ & $\operatorname{diag}(-1,1,1,1)$ \\
\hline 2 & $\operatorname{diag}(-1,-1,1,1)$ & $\operatorname{diag}(1,-1,1,1)$ \\
\hline 3 & $\operatorname{diag}(-1,1,-1,1)$ & $\operatorname{diag}(1,1,-1,1)$ \\
\hline 4 & $\operatorname{diag}(-1,1,1,-1)$ & $\operatorname{diag}(1,1,1,-1)$ \\
\hline 5 & $\operatorname{diag}(1,-1,-1,1)$ & $\operatorname{diag}(-1,-1,-1,1)$ \\
\hline 6 & $\operatorname{diag}(1,-1,1,-1)$ & $\operatorname{diag}(-1,-1,1,-1)$ \\
\hline 7 & $\operatorname{diag}(1,1,-1,-1)$ & $\operatorname{diag}(-1,1,-1,-1)$ \\
\hline 8 & $-\mathbf{1}_{4}$ & $\operatorname{diag}(1,-1,-1,-1)$ \\
\hline
\end{tabular}

Table 1: The $D$ matrices

where the $D$ matrices are given in Table 1 .

Since the final aim is to get the non-BPS boundary state in the GS formalism, we mention here precisely how one translates the above state in terms of the GS variables. From eqns. (3.19) and the mode expansions (2.9) we get the following:

$$
\begin{array}{ll}
J_{n}^{\mu \nu}=\frac{i}{4}\left(\gamma^{\mu} \bar{\gamma}^{\nu}\right)_{a b} \sum_{m \in \mathbf{Z}} \circ S_{m}^{a} S_{n-m^{\circ}}^{\circ} \quad \text { (IIB, IIA) }, \\
\tilde{J}_{n}^{\mu \nu}=\frac{i}{4}\left(\gamma^{\mu} \bar{\gamma}^{\nu}\right)_{a b} \sum_{m \in \mathbf{Z}} \circ \tilde{S}_{m}^{a} \tilde{S}_{n-m^{\circ}}^{b} \quad \text { (IIB) }, \\
\tilde{J}_{n}^{\mu \nu}=\frac{i}{4}\left(\bar{\gamma}^{\mu} \gamma^{\nu}\right)_{\dot{a} \dot{b} b} \sum_{m \in \mathbf{Z}} \circ \tilde{S}_{m}^{\dot{a}} \tilde{S}_{n-m^{\circ}}^{\circ} \quad \text { (IIA) . }
\end{array}
$$

The oscillator normal ordering used above is defined in the following way:

$$
{ }^{\circ} S_{m}^{a} S_{n}^{b \circ}=\left\{\begin{array}{rl}
S_{m}^{a} S_{n}^{b} & m \leq 0, \\
-S_{n}^{b} S_{m}^{a} & m>0 .
\end{array}\right.
$$

Then one uses eqns. (2.2), (2.3) and (5.32) to translate the $H_{-n}^{i}, E_{-n}^{\alpha_{i}}(i=1, \cdots, 4$, $n>0$ ) oscillators in terms of the GS variables. Since all the states in the expansion of the Ishibashi states (5.30) and (5.31) are defined in terms of these current modes and the current algebra vacuum states 20 in terms of the GS oscillators.

Let us now explain why we considered the "special alignments" to write down the explicit form of the Ishibashi states and in what sense they are special. In a formalism with

\footnotetext{
${ }^{20}$ These states in the lattice basis are defined in eqn. (A.4) in terms of the covariant basis vacuum states $|\mu\rangle$, which are in turn the same as the states in the representation of the fermionic zero mode oscillators (see the last paragraph of sec. 3).
} 
explicit covariance, no direction is special unless it is physically distinct. In the present case, it is the noncovariant nature of the basis construction which is responsible for this. As a result the Ishibashi states corresponding to the same world-volume dimension, but with different alignments may look very different, although they can be connected by some $S O(8)$ rotation. This is simply because the $\mathcal{T}$ operators for these two cases act very differently on the basis states due to the non-covariance. Let us consider the special subset of boundary conditions (4.1) for which the CSA oscillators $\left(H_{n}^{i}\right.$ 's) and the nonzero root oscillators $\left(E_{n}^{\alpha}\right)$ do not get mixed up by $\mathcal{T}$ (eqn. (4.4)). We will call a boundary condition of this type a CSA preserving boundary condition and the corresponding alignment a CSA preserving alignment. The special alignments correspond to boundary conditions of this type. Furthermore, the Ishibashi state for any CSA preserving alignment $\mathcal{C}$ can always be obtained from one of the specially aligned Ishibashi states by applying a rotation operator (which depends on $\mathcal{C}$ ), generated by $\tilde{H}_{0}^{i}$ 's or $\left(H_{0}^{i}+\tilde{H}_{0}^{i}\right)$ 's, on it. Such a rotation operator goes through the exponential operator in the eqn. (5.30) or (5.31) and acts on the bosonic vacuum states to give a phase $\varsigma^{(\mathcal{C})}(\vec{s}(\vec{n}))$. Therefore the general structure of an Ishibashi state with alignment $\mathcal{C}$, is the following:

$$
|\mathcal{C}, e\rangle\rangle=\exp \left[-\sum_{n>0} \frac{1}{n} H_{-n}^{T} D \tilde{H}_{-n}\right] \sum_{\vec{n}}^{\text {odd }} \varsigma^{(\mathcal{C})}(\vec{s}(\vec{n}))|\vec{s}(\vec{n})\rangle \otimes|\overline{-\vec{s}(D \vec{n})}\rangle
$$

where $D$ is one of the matrices given in Table 1 . This state can be rewritten in terms of the GS variables in the same way as discussed before.

The rotation operators required for generating all the CSA preserving alignments from the special alignments given in eqns. (5.28), (5.29) are,

$$
\begin{aligned}
\mathcal{U}_{i} & \equiv e^{i \pi \tilde{H}_{0}^{i}} \\
\mathcal{R}_{i}\left(\theta_{i}\right) & \equiv e^{i \theta_{i}\left(H_{0}^{i}+\tilde{H}_{0}^{i}\right)} .
\end{aligned}
$$

Notice that $\mathcal{R}_{i}\left(\theta_{i}\right)$ acts both on the right and left parts and hence gives a physical rotation to a brane by an angle $\theta_{i}$ on the $(2 i-1)-2 i$ plane. But since $\mathcal{U}_{i}$ acts only on the right part, generically, acting on some state this gives another physically inequivalent state (state which is not connected to the initial one through any symmetry transformation) in the Hilbert space ${ }^{22}$. $\mathcal{U}_{i}$ provides the reflection on the coordinates $\mu=2 i-1,2 i$. Acting on an Ishibashi state which is not aligned along the $(2 i-1)-2 i$ plane, $\mathcal{U}_{i}$ extends its world-volume to this additional plane and similarly acting on an Ishibashi state already aligned along the $(2 i-1)-2 i$ plane, it reduces its world-volume by this plane. If the initial Ishibashi state has one of the two directions $\mu=2 i-1,2 i$ to be tangential and the other normal, then $\mathcal{U}_{i}$ interchanges these tangential and normal directions. But this is same as performing a

\footnotetext{
${ }^{21}$ It has been mentioned in sec. 1 that to make the basis, constructed in terms of currents, free of null states, one has to break the explicit covariance.

${ }^{22}$ There are exceptions which we are going to discuss below.
} 
physical rotation on the $(2 i-1)-2 i$ plane through $\pi / 2$ which could also be achieved by applying $\mathcal{R}_{i}(\pi / 2)$. Given all these, following will be the prescription for generating the whole class of Ishibashi states starting from that of the corresponding special alignment: Take the Ishibashi state of the special alignment and operate only those $\mathcal{U}_{i}$ and $\mathcal{R}_{i}\left(\theta_{i}\right)$ operators on it to get the whole class of Ishibashi states, which are enlisted in Tables 2 and 3. The $\mathcal{U}_{i}$ operators which are excluded from the list give over-counting, because the same state is obtained by applying $\mathcal{R}_{i}(\pi / 2)$. The $\mathcal{R}_{i}\left(\theta_{i}\right)$ operators that have been excluded from the list have trivial action on the initial state, because they provide rotations on the planes which are either orthogonal or tangential to the initial state.

Now let us discuss the other alignments which do not preserve the CSA space. An Ishibashi state corresponding to this type of boundary condition will look considerably different from those discussed above. In this case, one again starts from the eqn. similar to $(5.20)$, but now with the following general form of $\mathcal{T}$ :

$$
\mathcal{T}=\exp \left[i f\left(H_{0}^{i}, E_{0}^{\alpha}\right)\right],
$$

where $f\left(H_{0}^{i}, E_{0}^{\alpha}\right)$ is a linear function of its arguments such that $\mathcal{T}$ is hermitian (because $\mathcal{T}$ is unitary and also squares to identity in the vector representation $\left.\Pi^{(e)}\right)$. We can treat this type of Ishibashi states in the following way: Any Ishibashi state $|f, e\rangle\rangle$ with CSA non-preserving boundary condition corresponding to the function $f\left(H_{0}^{i}, E_{0}^{\alpha}\right)$, can be written in the following form:

$$
|f, e\rangle\rangle=\mathcal{O}(f, \mathcal{C})|\mathcal{C}, e\rangle\rangle
$$

where the state $|\mathcal{C}, e\rangle\rangle$ is defined in eqn. (5.34) and $\mathcal{O}(f, \mathcal{C})$ is an $S O(8)$ rotation operator which acts both on the right and the left parts. For a given function $f\left(H_{0}^{i}, E_{0}^{\alpha}\right)$, the rotation operator $\mathcal{O}$ depends on the specific CSA preserving alignment $\mathcal{C}$ chosen on the right hand side of the above eqn. To get the state $|f, e\rangle\rangle$ in GS formalism one can first translate the state $|\mathcal{C}, e\rangle\rangle$ in terms of the GS variables (following the procedure that has already been discussed), then apply the known rotation operator $\mathcal{O}(f, \mathcal{C})$ (under which the GS variables transform covariantly) on that. 


\begin{tabular}{|c|c|c|c|}
\hline \hline$\rho$ & $N_{\rho}^{A}$ & $\mathcal{U}_{i}$ & $\mathcal{R}_{i}\left(\theta_{i}\right)$ \\
\cline { 3 - 4 } & & $i$ & $i$ \\
\hline \hline 1 & $\{$ null $\}$ & $1,2,3,4$ & - \\
\hline 2 & $\{2,4\}$ & 3,4 & 1,2 \\
\hline 3 & $\{2,6\}$ & 2,4 & 1,3 \\
\hline 4 & $\{2,8\}$ & 2,3 & 1,4 \\
\hline 5 & $\{4,6\}$ & 1,4 & 2,3 \\
\hline 6 & $\{4,8\}$ & 1,3 & 2,4 \\
\hline 7 & $\{6,8\}$ & 1,2 & 3,4 \\
\hline 8 & $\{2,4,6,8\}$ & - & $1,2,3,4$ \\
\hline
\end{tabular}

Table 2: Allowed $\mathcal{U}_{i}$ and $\mathcal{R}_{i}\left(\theta_{i}\right)$ operators for $\left\{N_{\rho}^{A}\right\}$.

\begin{tabular}{|c|c|c|c|}
\hline \hline$\rho$ & $N_{\rho}^{B}$ & $\mathcal{U}_{i}$ & $\mathcal{R}_{i}\left(\theta_{i}\right)$ \\
\cline { 3 - 4 } & & $i$ & $i$ \\
\hline \hline 1 & $\{2\}$ & $2,3,4$ & 1 \\
\hline 2 & $\{4\}$ & $1,3,4$ & 2 \\
\hline 3 & $\{6\}$ & $1,2,4$ & 3 \\
\hline 4 & $\{8\}$ & $1,2,3$ & 4 \\
\hline 5 & $\{2,4,6\}$ & 4 & $1,2,3$ \\
\hline 6 & $\{2,4,8\}$ & 3 & $1,2,4$ \\
\hline 7 & $\{2,6,8\}$ & 2 & $1,3,4$ \\
\hline 8 & $\{4,6,8\}$ & 1 & $2,3,4$ \\
\hline
\end{tabular}

Table 3: Allowed $\mathcal{U}_{i}$ and $\mathcal{R}_{i}\left(\theta_{i}\right)$ operators for $\left\{N_{\rho}^{B}\right\}$. 


\section{A Vacuum Representations}

Here we will give the explicit vacuum representations $\Pi_{0}^{(w)}$ for $w=0, e, \bar{\delta}, \delta$.

1. $\Pi_{0}^{(0)}$ :

This contains only one state $|0\rangle$ defined by:

$$
L_{0}^{S U G}|0\rangle=J_{n}^{\mu \nu}|0\rangle=0, \quad \forall n \geq 0 .
$$

This is the $S L(2, \mathbf{C})$ and $S O(8)$ invariant vacuum state.

2. $\Pi_{0}^{(e)}$ :

The orthonormal states are denoted $|\mu\rangle, \quad \mu=1,2, \ldots, 8$ and the representation is given by (see eqn. (2.17)):

$$
T_{(e)}^{\mu \nu}=i \Sigma^{\mu \nu}
$$

where $\Sigma^{\mu \nu}$ is an $8 \times 8$ matrix, elements of which are given by:

$$
\left(\Sigma^{\mu \nu}\right)_{\rho \sigma}=\delta^{\mu \rho} \delta^{\nu \sigma}-\delta^{\mu \sigma} \delta^{\nu \rho} .
$$

Now define states in the lattice basis as,

$$
\left| \pm e_{j}\right\rangle \equiv \frac{1}{\sqrt{2}}(|\mu=2 j-1\rangle \mp i|\mu=2 j\rangle) \text {. }
$$

From the representation of $J_{0}^{\mu \nu}$ given above (eqns. (2.17), (A.2)), one can find the representations for the $H_{0}^{i}$, s in $\Pi_{0}^{(e)}$ by using eqn. (2.2). From this the $H_{0}^{i}=P^{i}$ eigenvalues of the states $\left| \pm e_{j}\right\rangle$ can be computed. One finds,

$$
P\left| \pm e_{i}\right\rangle= \pm e_{i}\left| \pm e_{i}\right\rangle
$$

where $e_{i}$ 's are weight vectors defined in eqns. (2.4). Using eqn. (2.3), the matrices for $E_{0}^{\alpha}$ can also be computed. One can show the following relations:

$$
\begin{aligned}
& \left|-e_{1}\right\rangle=E_{0}^{-\alpha_{1}} E_{0}^{-\alpha_{2}}\left|e_{1}\right\rangle, \\
& \left|e_{2}\right\rangle=i E_{0}^{-\alpha_{2}}\left|e_{1}\right\rangle, \quad\left|-e_{2}\right\rangle=i E_{0}^{-\alpha_{1}}\left|e_{1}\right\rangle, \\
& \left|e_{3}\right\rangle=i E_{0}^{-\alpha_{6}}\left|e_{1}\right\rangle, \quad\left|-e_{3}\right\rangle=i E_{0}^{-\alpha_{5}}\left|e_{1}\right\rangle, \\
& \left|e_{4}\right\rangle=i E_{0}^{-\alpha_{8}}\left|e_{1}\right\rangle, \quad\left|-e_{4}\right\rangle=i E_{0}^{-\alpha_{7}}\left|e_{1}\right\rangle . \\
& \left|e_{1}\right\rangle=E_{0}^{\alpha_{1}} E_{0}^{\alpha_{2}}\left|-e_{1}\right\rangle, \\
& \left|e_{2}\right\rangle=i E_{0}^{\alpha_{1}}\left|-e_{1}\right\rangle, \quad\left|-e_{2}\right\rangle=i E_{0}^{\alpha_{2}}\left|-e_{1}\right\rangle, \\
& \left|e_{3}\right\rangle=i E_{0}^{\alpha_{5}}\left|-e_{1}\right\rangle, \quad\left|-e_{3}\right\rangle=i E_{0}^{\alpha_{6}}\left|-e_{1}\right\rangle, \\
& \left|e_{4}\right\rangle=i E_{0}^{\alpha_{7}}\left|-e_{1}\right\rangle, \quad\left|-e_{4}\right\rangle=i E_{0}^{\alpha_{8}}\left|-e_{1}\right\rangle .
\end{aligned}
$$


3. $\Pi_{0}^{(\bar{\delta})}$ :

The orthonormal states are denoted $|\dot{a}\rangle, \quad \dot{a}=1,2, \ldots, 8$ and the representation is given by:

$$
T_{(\bar{\delta})}^{\mu \nu}=\frac{i}{2} \bar{\gamma}^{\mu} \gamma^{\nu} .
$$

(For definition and explicit reprsentation of the $8 \times 8$ matrices $\gamma^{\mu}$ and $\bar{\gamma}^{\mu}$, see appendix $B$.) The states in the lattice basis are:

$$
\left| \pm \bar{\delta}_{j}\right\rangle \equiv \frac{1}{\sqrt{2}}(|\dot{a}=2 j-1\rangle \mp i|\dot{a}=2 j\rangle),
$$

where $\bar{\delta}_{j}$ 's are the following four conjugate spinor weights:

$$
\bar{\delta}_{1}=\frac{1}{2}\left(\begin{array}{c}
1 \\
1 \\
-1 \\
1
\end{array}\right), \bar{\delta}_{2}=\frac{1}{2}\left(\begin{array}{c}
1 \\
1 \\
1 \\
-1
\end{array}\right), \bar{\delta}_{3}=\frac{1}{2}\left(\begin{array}{c}
-1 \\
1 \\
1 \\
1
\end{array}\right), \bar{\delta}_{4}=\frac{1}{2}\left(\begin{array}{c}
-1 \\
1 \\
-1 \\
-1
\end{array}\right) .
$$

Following the same procedure as described for eqn. (A.5) one can establish the following eigenvalue equation for the present representation:

$$
P\left| \pm \bar{\delta}_{i}\right\rangle= \pm \bar{\delta}_{i}\left| \pm \bar{\delta}_{i}\right\rangle .
$$

Similarly as before one can check the following relations:

$$
\begin{aligned}
\left|-\bar{\delta}_{1}\right\rangle=-i E_{0}^{-\alpha_{1}}\left|\bar{\delta}_{2}\right\rangle, & \left|\bar{\delta}_{1}\right\rangle=E_{0}^{-\alpha_{4}}\left|\bar{\delta}_{2}\right\rangle, \\
\left|-\bar{\delta}_{2}\right\rangle=i E_{0}^{-\alpha_{1}} E_{0}^{-\alpha_{4}}\left|\bar{\delta}_{2}\right\rangle, & \left|\bar{\delta}_{3}\right\rangle=-E_{0}^{-\alpha_{8}}\left|\bar{\delta}_{2}\right\rangle, \\
\left|-\bar{\delta}_{3}\right\rangle=i E_{0}^{-\alpha_{9}}\left|\bar{\delta}_{2}\right\rangle, & \left|\bar{\delta}_{4}\right\rangle=-i E_{0}^{-\alpha_{5}}\left|\bar{\delta}_{2}\right\rangle \\
\left|-\bar{\delta}_{4}\right\rangle=-E_{0}^{-\alpha_{12}}\left|\bar{\delta}_{2}\right\rangle, & \\
\left|\bar{\delta}_{1}\right\rangle=-i E_{0}^{\alpha_{1}}\left|-\bar{\delta}_{2}\right\rangle, & \left|-\bar{\delta}_{1}\right\rangle=-E_{0}^{\alpha_{4}}\left|-\bar{\delta}_{2}\right\rangle, \\
\left|\bar{\delta}_{2}\right\rangle=-i E_{0}^{\alpha_{1}} E_{0}^{\alpha_{4}}\left|-\bar{\delta}_{2}\right\rangle, & \\
\left|\bar{\delta}_{3}\right\rangle=i E_{0}^{\alpha_{9}}\left|-\bar{\delta}_{2}\right\rangle, & \left|-\bar{\delta}_{3}\right\rangle=E_{0}^{\alpha_{8}}\left|-\bar{\delta}_{2}\right\rangle \\
\left|\bar{\delta}_{4}\right\rangle=E_{0}^{\alpha_{12}}\left|-\bar{\delta}_{2}\right\rangle, & \left|-\bar{\delta}_{4}\right\rangle=-i E_{0}^{\alpha_{5}}\left|-\bar{\delta}_{2}\right\rangle .
\end{aligned}
$$

4. $\Pi_{0}^{(\delta)}$ :

The orthonormal states are denoted $|a\rangle, \quad a=1,2, \ldots, 8$ and the representation is given by:

$$
T_{(\delta)}^{\mu \nu}=\frac{i}{2} \gamma^{\mu} \bar{\gamma}^{\nu} .
$$


The states in the lattice basis are:

$$
\left| \pm \delta_{j}\right\rangle \equiv \frac{1}{\sqrt{2}}(|a=2 j-1\rangle \mp i|a=2 j\rangle),
$$

where $\delta_{j}$ 's are the following four spinor weights:

$$
\delta_{1}=\frac{1}{2}\left(\begin{array}{c}
-1 \\
1 \\
-1 \\
1
\end{array}\right), \delta_{2}=\frac{1}{2}\left(\begin{array}{c}
-1 \\
1 \\
1 \\
-1
\end{array}\right), \delta_{3}=\frac{1}{2}\left(\begin{array}{l}
1 \\
1 \\
1 \\
1
\end{array}\right), \delta_{4}=\frac{1}{2}\left(\begin{array}{c}
1 \\
1 \\
-1 \\
-1
\end{array}\right)
$$

Proceeding in the same way as before one can derive the following equation for the present representation:

$$
P\left| \pm \delta_{i}\right\rangle= \pm \delta_{i}\left| \pm \delta_{i}\right\rangle
$$

Again the following relations are true:

$$
\begin{array}{rlrl}
\left|-\delta_{1}\right\rangle & =E_{0}^{-\alpha_{11}}\left|\delta_{3}\right\rangle, & & \left|\delta_{1}\right\rangle=i E_{0}^{-\alpha_{5}}\left|\delta_{3}\right\rangle, \\
\left|-\delta_{2}\right\rangle=-i E_{0}^{-\alpha_{9}}\left|\delta_{3}\right\rangle, & & \left|\delta_{2}\right\rangle=E_{0}^{-\alpha_{7}}\left|\delta_{3}\right\rangle \\
\left|-\delta_{3}\right\rangle=i E_{0}^{-\alpha_{1}} E_{0}^{-\alpha_{3}}\left|\delta_{3}\right\rangle, & & \\
\left|-\delta_{4}\right\rangle & =-i E_{0}^{-\alpha_{1}}\left|\delta_{3}\right\rangle, & & \left|\delta_{4}\right\rangle=E_{0}^{-\alpha_{3}}\left|\delta_{3}\right\rangle \\
\left|\delta_{1}\right\rangle=-E_{0}^{\alpha_{11}}\left|-\delta_{3}\right\rangle, & \left|-\delta_{1}\right\rangle=i E_{0}^{\alpha_{5}}\left|-\delta_{3}\right\rangle \\
\left|\delta_{2}\right\rangle=-i E_{0}^{\alpha_{9}}\left|-\delta_{3}\right\rangle, & \left|-\delta_{2}\right\rangle=-E_{0}^{\alpha_{7}}\left|-\delta_{3}\right\rangle \\
\left|\delta_{3}\right\rangle=-i E_{0}^{\alpha_{1}} E_{0}^{\alpha_{3}}\left|-\delta_{3}\right\rangle, & \\
\left|\delta_{4}\right\rangle=-i E_{0}^{\alpha_{1}}\left|-\delta_{3}\right\rangle, & \left|-\delta_{4}\right\rangle=-E_{0}^{\alpha_{3}}\left|-\delta_{3}\right\rangle
\end{array}
$$

\section{B $\Gamma$-Matrix Representation}

Let $\Gamma^{\mu}(\mu=1,2, \ldots, 8)$ be the 16 -dimensional $S O(8)$ Dirac matrices satisfying the Clifford algebra:

$$
\left\{\Gamma^{\mu}, \Gamma^{\nu}\right\}=2 \delta^{\mu \nu} \mathbf{1}_{16}
$$

$\mathbf{1}_{16}=(16 \times 16)$ identity matrix. In the real-Weyl basis they take the following block-offdiagonal form:

$$
\Gamma^{\mu}=\left(\begin{array}{cc}
0 & \gamma^{\mu} \\
\bar{\gamma}^{\mu} & 0
\end{array}\right)
$$

where $\gamma^{\mu}$ are $(8 \times 8)$ real matrices with $\bar{\gamma}^{\mu}=\left(\gamma^{\mu}\right)^{T}$. In terms of $\gamma^{\mu}$ 's, the algebra (B.1) reads:

$$
\gamma^{\mu} \bar{\gamma}^{\nu}+\gamma^{\nu} \bar{\gamma}^{\mu}=\bar{\gamma}^{\mu} \gamma^{\nu}+\bar{\gamma}^{\nu} \gamma^{\mu}=2 \delta^{\mu \nu} \mathbf{1}_{8}
$$


$\mathbf{1}_{8}=(8 \times 8)$ identity matrix. We take the following representation of the $\gamma$ matrices [10].

$$
\begin{array}{ll}
\gamma^{1}=\mathbf{1} \times \mathbf{1} \times \mathbf{1}, & \gamma^{2}=\epsilon \times \mathbf{1} \times \sigma_{3}, \\
\gamma^{3}=\sigma_{3} \times \epsilon \times \mathbf{1}, & \gamma^{4}=\sigma_{1} \times \epsilon \times \mathbf{1}, \\
\gamma^{5}=\mathbf{1} \times \sigma_{3} \times \epsilon, & \gamma^{6}=\epsilon \times \mathbf{1} \times \sigma_{1}, \\
\gamma^{7}=\epsilon \times \epsilon \times \epsilon, & \gamma^{8}=\mathbf{1} \times \sigma_{1} \times \epsilon,
\end{array}
$$

where $\mathbf{1}=(2 \times 2)$ identity matrix, $\epsilon=i \sigma_{2}$ and $\sigma_{1}, \sigma_{2}, \sigma_{3}$ are the Pauli matrices:

$$
\sigma_{1}=\left(\begin{array}{ll}
0 & 1 \\
1 & 0
\end{array}\right), \quad \sigma_{2}=\left(\begin{array}{cc}
0 & -i \\
i & 0
\end{array}\right), \quad \sigma_{3}=\left(\begin{array}{cc}
1 & 0 \\
0 & -1
\end{array}\right)
$$

\section{Norm of Bosonic Vacuum states}

Here we will show that the norm of the bosonic vacuum states $\left|\vec{s} \in \mathcal{K}^{(e)}\right\rangle$, defined in eqn. (5.5), with respect to hermitian inner product is 1 . From eqn. (5.5) we can write,

$$
\begin{aligned}
|| \vec{s}\rangle\left.\right|^{2} \equiv & \langle\vec{s} \mid \vec{s}\rangle \\
= & \left\langle V\left|\prod_{i}\left\{\mathcal{E}^{\alpha_{i}}\left(s_{i}\right)\right\}^{\dagger} \prod_{j} \mathcal{E}^{\alpha_{j}}\left(s_{j}\right)\right| V\right\rangle \\
= & \langle V|\left\{\mathcal{E}^{\alpha_{4}}\left(s_{4}\right)\right\}^{\dagger} \cdots\left\{\mathcal{E}^{\alpha_{1}}\left(s_{1} \mp 1\right)\right\}^{\dagger}\left(E_{2\left|s_{1}\right|-1}^{\mp \alpha_{1}} E_{-2\left|s_{1}\right|+1}^{ \pm \alpha_{1}}\right) \\
& \mathcal{E}^{\alpha_{1}}\left(s_{1} \mp 1\right) \cdots \mathcal{E}^{\alpha_{4}}\left(s_{4}\right)|V\rangle
\end{aligned}
$$

where the upper and lower signs of \pm or $\mp$ used above corresponds to the fact that $s_{1}>0$ and $s_{1}<0$ respectively. The same notation will be followed throughout the rest of the derivation. Now using the commutator between $E_{2\left|s_{1}\right|-1}^{\mp \alpha_{1}}$ and $E_{-2\left|s_{1}\right|+1}^{ \pm \alpha_{1}}$ (see eqn. (2.7)) we can write:

$$
|| \vec{s}\rangle\left.\right|^{2}=I_{1}+I_{2}
$$

where,

$$
\begin{aligned}
& I_{1}=\left\langle s_{1} \mp 1, s_{2}, s_{3}, s_{4}\left|\left(\mp \alpha_{1} \cdot H_{0}+2\left|s_{1}\right|-1\right)\right| s_{1} \mp 1, s_{2}, s_{3}, s_{4}\right\rangle, \\
& \left.I_{2}=\left|E_{2\left|s_{1}\right|-1}^{\mp \alpha_{1}}\right| s_{1} \mp 1, s_{2}, s_{3}, s_{4}\right\rangle\left.\right|^{2} .
\end{aligned}
$$

For $I_{1}$, one uses the orthogonality of $\alpha_{i}$ 's and the fact that $\alpha_{i}^{2}=2$ to get,

$$
\begin{aligned}
I_{1} & \left.=\left(1+2\left|s_{1}\right| \mp 2 s_{1}\right)|| s_{1} \mp 1, s_{2}, s_{3}, s_{4}\right\rangle\left.\right|^{2}, \\
& \left.=|| s_{1} \mp 1, s_{2}, s_{3}, s_{4}\right\rangle\left.\right|^{2},
\end{aligned}
$$


since according to the rule mentioned above, $\mp 2 s_{1}=-2\left|s_{1}\right|$.

For $I_{2}$, one may proceed as follows:

$$
\begin{aligned}
& E_{2\left|s_{1}\right|-1}^{\mp \alpha_{1}}\left|s_{1} \mp 1, s_{2}, s_{3}, s_{4}\right\rangle \\
&= E_{2\left|s_{1}\right|-1}^{\mp \alpha_{1}} E_{-2\left|s_{1}\right|+3}^{ \pm \alpha_{1}}\left|s_{1} \mp 2, s_{2}, s_{3}, s_{4}\right\rangle, \\
&= \mp \alpha_{1} . H_{2}\left|s_{1} \mp 2, s_{2}, s_{3}, s_{4}\right\rangle+E_{-2\left|s_{1}\right|+3}^{ \pm \alpha_{1}} E_{2\left|s_{1}\right|-1}^{\mp \alpha_{1}}\left|s_{1} \mp 2, s_{2}, s_{3}, s_{4}\right\rangle, \\
& {[\text { see eqn. }(2.7)] } \\
&= 0+E_{-2\left|s_{1}\right|+3}^{ \pm \alpha_{1}} E_{2\left|s_{1}\right|-1}^{\mp \alpha_{1}} E_{-2\left|s_{1}\right|+5}^{ \pm \alpha_{1}}\left|s_{1} \mp 3, s_{2}, s_{3}, s_{4}\right\rangle, \\
&= E_{-2\left|s_{1}\right|+3}^{ \pm \alpha_{1}}\left(\mp \alpha_{1} . H_{4}\right)\left|s_{1} \mp 3, s_{2}, s_{3}, s_{4}\right\rangle+ \\
& E_{-2\left|s_{1}\right|+3}^{ \pm \alpha_{1}} E_{-2\left|s_{1}\right|+5}^{ \pm \alpha_{1}} E_{2\left|s_{1}\right|-1}^{\mp \alpha_{1}}\left|s_{1} \mp 3, s_{2}, s_{3}, s_{4}\right\rangle \\
&= 0+E_{-2\left|s_{1}\right|+3}^{ \pm \alpha_{1}} E_{-2\left|s_{1}\right|+5}^{ \pm \alpha_{1}} E_{2\left|s_{1}\right|-1}^{\mp \alpha_{1}}\left|s_{1} \mp 3, s_{2}, s_{3}, s_{4}\right\rangle . \\
& {[\text { again using eqn. (5.2)] }}
\end{aligned}
$$

Proceeding in the same way one finally gets,

$$
E_{2\left|s_{1}\right|-1}^{\mp \alpha_{1}}\left|s_{1} \mp 1, s_{2}, s_{3}, s_{4}\right\rangle=\mathcal{E}^{\alpha_{1}}\left(s_{1} \mp 1\right) E_{2\left|s_{1}\right|-1}^{\mp \alpha_{1}} \prod_{i=2}^{4} \mathcal{E}^{\alpha_{i}}\left(s_{i}\right)|V\rangle .
$$

But since all $E_{n}^{\alpha_{i}}$ 's commute, $E_{2\left|s_{1}\right|-1}^{\mp \alpha_{1}}$ in the above equation passes through all the $\mathcal{E}^{\alpha_{i}}\left(s_{i}\right)^{\prime}$ s, hits the vacuum state $|V\rangle$ and annihilates it (as $2\left|s_{1}\right|-1>0$ ). Therefore,

$$
I_{2}=0 \text {. }
$$

Therefore, using eqns. (C.2), (C.4) and (C.6) we get,

$$
\left.|| \vec{s}\rangle\left.\right|^{2}=|| s_{1} \mp 1, s_{2}, s_{3}, s_{4}\right\rangle\left.\right|^{2} \text {. }
$$

Repeating the above procedure to remove all the $E$ oscilltors sandwiched between $\langle V|$ and $|V\rangle$, one can finally show:

$$
\left.|| \vec{s}\rangle\left.\right|^{2}=|| V\right\rangle\left.\right|^{2}=1
$$

\section{Acknowledgements}

I am extremely grateful to Ashoke Sen for suggesting the problem. His guidance and enormous help throughout the process of solving the problem and writing the paper has made this work possible. I would like to thank Debashis Ghoshal and Dileep P. Jatkar for their patient and critical reading of the preliminary draft. 


\section{References}

[1] J. Dai, R. Leigh, J. Polchinski, Mod. Phys. Lett.A4 (1989) 2073 ;

R. Leigh, Mod. Phys. Lett. A4 (1989) 2767 ;

J. Polchinski, S. Chaudhuri and C. V. Johnson,

"Notes on D-Branes", hep-th/9602052;

J. Polchinski, "TASI lectures on D-Branes", hep-th/9611050.

[2] Paolo Di Vecchia, Antonella Liccardo, "D branes in string theory, I", hep-th/9912161;

Paolo Di Vecchia, Antonella Liccardo, "D branes in string theory, II", hepth/9912275.

[3] O. Bergman, M. R. Gaberdiel, "Stable non-BPS D-particles", Phys. Lett. B441 (1988) 133, hep-th/9806155.

[4] A. Sen, "Type I D-particle and its interactions", JHEP 10 (1989) 021, hepth/9809111.

[5] A. Sen, "BPS D-branes on non-supersymmetric cycles, JHEP 12 (1998) 021, hepth/9812031.

[6] A. Sen, "Non-BPS States and Branes in String Theory", hep-th/9904207.

[7] M. R. Gaberdiel, "Lectures on non-BPS Dirichlet branes", Class. Quant. Grav. 17 (2000) 3483, hep-th/0005029.

[8] J. Polchinski, "String Theory", Vol. 1.

[9] J. Polchinski, "String Theory", Vol. 2.

[10] M. Green, J. Schwarz, E. Witten, "Superstring Theory" Vol. 1.

[11] E. Witten, "D = 10 superstring theory", in Fourth Workshop on Grand Unification, ed. P. Langacker et al. (Birkhauser), (1983) p. 395.

[12] C. G. Callan, C. Lovelace, C. R. Nappi, S. A. Yost, "Adding holes and crosscaps to the superstring", Nucl. Phys. B293 (1987) 83 ;

J. Polchinski, Y. Cai, "Consistency of open superstring theories", Nucl. Phys. B296 (1988) 91 ;

C. G. Callan, C. Lovelace, C. R. Nappi, S. A. Yost, "Loop corections to superstring equations of motion", Nucl. Phys.B308 (1988) 221 ;

M. Li, "Boundary states of D-branes and Dy-strings", Nucl. Phys. B460 (1996) 351, hep-th/9510161;

M. Frau, L. Gallot, A. Lerda, P. Strigazzi, "Stable non-BPS D-branes in type-I string theory", Nucl. Phys. B564 (2000) 60, hep-th/9903123. 
[13] O. Bergman, M. R. Gaberdiel, "A non-supersymmetric open string theory and Sduality", Nucl. Phys. B499 (1997) 183, hep-th/9701137;

A. Sen, "Stable non-BPS bound states of BPS D-branes" JHEP 08 (1998) 010, hepth/9805019.

[14] M. B. Green, "Point-like states for type 2b superstrings", Phys. Lett. B329 (1994) 435 .

[15] M. B. Green, M. Gutperle, "Light-cone Supersymmetry and D-Branes", Nucl. Phy. B476 (1996) 484, hep-th/9604091.

[16] H. Georgi, "Lie algebras in particle physics" (1982), Benjamin/Cummings.

[17] P. Goddard, D. Olive, "Kac-Moody and Virasoro algebras in relation to quantum physics", Int. J. Mod. Phys. A1 (1986), 303.

[18] J. Fuchs, C. Schweigert, "Symmetries, Lie Algebras and Representations." (1997) Cambridge University Press ;

Philippe Di Francesco, Pierre Mathieu, David Senechal, "Conformal Field Theory", Springer-Verlag.

[19] N. Ishibashi, "The Boundary and Crosscap States in Conformal Field Theories", Mod. Phys. Lett. A4 (1989) 251 ;

T. Onogi, N. Ishibashi, "Conformal field theories on surfaces with boundaries and crpsscaps", Mod. Phys. Lett. A4 (1989) 161 ;

J. L. Cardy, "Boundary conditions, fusion rules and the Verlinde formula", Nucl. Phys. B324 (1989) 581;

A. Recknagel, V. Schomerus, "D-branes in Gepner models", Nucl. Phys. B531 (1998) 185, hep-th/9712186 ;

S. Stanciu, "D-branes in group manifolds", JHEP 01 (2000) 025, hep-th/9909163;

H. Ishikawa, S. Watamura, "Free field realization of D-branes in group manifold", JHEP 08 (2000) 044, hep-th/0007141.

[20] M. Kato, T. Okada, "D-branes on group manifolds", Nucl. Phys. B499 (1997) 583, hep-th/9612148.

[21] D. Friedan, E. Martinec, S. Shenker, "Conformal invariance, supersymmetry, and string theory", Nucl. Phys. B271 (1986) 93 ;

"BOSONIZATION" ed. Michael Stone, World Scientific. 